Comment. Math. Helv. 76 (2001) 110-154

(C) 2001 Birkhäuser Verlag, Basel

Commentarii Mathematici Helvetici

\title{
Central extensions of Lie superalgebras
}

Kenji Iohara and Yoshiyuki Koga

\begin{abstract}
For a commutative algebra A over a commutative ring $k$ satisfying certain conditions, we construct the universal central extension of $\mathfrak{g}_{k} \otimes_{k} \mathrm{~A}$, regarded as a Lie superalgebra over $k$, where $\mathfrak{g}_{k}$ denotes a basic classical Lie superalgebra over $k$. To consider basic classical Lie superalgebras over an ring $k$, we also show the existence of their Chevalley basis. Our results contain not only the descriptions of the untwisted affine Lie superalgebras but also those of the toroidal Lie superalgebras.
\end{abstract}

Mathematics Subject Classification (2000). 17B05, 17B55, $19 \mathrm{D} 55$.

Keywords. Lie superalgebras, Central extensions, Cyclic homology.

\section{Introduction}

The universal central extensions (UCE for short) of a simple Lie algebra over commutative rings were studied in [Gar] and [KL]. To be precise, let $\mathfrak{g}$ be a simple finite dimensional Lie algebra over the field of complex numbers $\mathbb{C}$. It is known that the UCE of the Lie algebra $\mathfrak{g} \otimes \mathbb{C}\left[t^{ \pm 1}\right]$ is a one-dimensional central extension, and this is just an affine Lie algebra [Gar]. More generally, let A be a commutative algebra over a commutative ring $k$ satisfying $\frac{1}{2} \in k$, and let $\mathfrak{g}_{k}$ be a simple finite dimensional Lie algebra over $k$, in the sense of [Che]. Then the kernel of the UCE of the Lie algebra $\mathfrak{g}_{k} \otimes \mathrm{A}$ is $\Omega_{\mathrm{A} / k}^{1} / d \mathrm{~A}$, the A -module of Kähler differentials over $k$ modulo exact forms, which was obtained by C. Kassel and J.-L. Loday [KL] (see also [Kas]).

Representation theories of affine Lie superalgebras have been developed recently e.g. $[\mathrm{KW}$. Moreover, the Serre relations of affine Lie superalgebras were obtained by $\mathrm{H}$. Yamane [Ya]. Although it might be known to the experts, it seems that there is no literature in which an affine Lie superalgebra is realized as the UCE of the Lie superalgebra $\mathfrak{g} \otimes \mathbb{C}\left[t^{ \pm 1}\right]$, where $\mathfrak{g}$ is the underlying finite dimensional simple Lie superalgebra. In this paper, we address ourselves to the description of the UCE of the Lie superalgebra $\mathfrak{g}_{k} \otimes_{k} \mathrm{~A}$ in the case when $\mathfrak{g}_{k}$ is a basic classical Lie superalgebra, viz. a Lie superalgebra which has a non-degenerate, even, supersymmetric, invariant bilinear form, classified by Kac [Kac1], [Kac2]. Namely, we 
first construct a $\mathbb{Z}$-basis of $\mathfrak{g}$ à la Chevalley [Che] (Theorem 3.9), and define the Lie superalgebra $\mathfrak{g}_{k}$. After that, we compute the UCE of the Lie superalgebra $\mathfrak{g}_{k} \otimes \mathrm{A}$.

The main result of this paper can be stated as follows (Theorem 4.7): Let $k$ be a commutative ring satisfying certain conditions, and let $\mathrm{A}$ be a commutative algebra over $k$. Let $\mathfrak{g}$ be a basic classical Lie superalgebra. Then the UCE $\mathfrak{g}_{k}(\mathrm{~A})$ of $\mathfrak{g}_{k} \otimes \mathrm{A}$ is given by

$$
\mathfrak{g}_{k}(\mathrm{~A}) \simeq\left\{\begin{array}{cl}
\mathfrak{g}_{k} \otimes \mathrm{A} \oplus \Omega_{\mathrm{A} / k}^{1} / d \mathrm{~A} & \text { if } \mathfrak{g} \text { is not of type } \mathrm{A}(n, n) \forall n \\
\mathfrak{d}_{k} \otimes \mathrm{A} \oplus \Omega_{\mathrm{A} / k}^{1} / d \mathrm{~A} & \text { if } \mathfrak{g} \text { is of type } \mathrm{A}(1,1) \\
\mathfrak{s l}(n+1, n+1)_{k} \otimes \mathrm{A} \oplus \Omega_{\mathrm{A} / k}^{1} / d \mathrm{~A} & \text { if } \mathfrak{g} \text { is of type } \mathrm{A}(n, n) \exists n>1
\end{array}\right.
$$

where $\mathfrak{d}$ is the Lie superalgebra of type " $\mathrm{D}(2,1,-1)$ " (see $\S 2.4)$. In particular, for $k:=\mathbb{C}$ and $\mathrm{A}:=\mathbb{C}\left[t^{ \pm 1}\right]$, if $\mathfrak{g}$ is not of type $\mathrm{A}(n, n)$ for any $n$, then the $\mathrm{UCE}$ is just the one-dimensional central extension as in the non-super case.

Let us explain our approach to the proof of Theorems 3.9 and 4.7. Basically, we follow the lines due to [Che] and [Gar]. That is, we first consider the rank 1 super-subalgebras of a basic classical Lie superalgebra, i.e., $\mathfrak{s l}_{2}, \mathfrak{o s p}(1,2)$ and $\mathfrak{s l}(1,1)$, and then we consider the rank 2 subalgebras. But technical difficulties arise owing to the following reasons:

1. There exists roots of length 0 .

2. $\mathfrak{s l}(1,1)$ is not perfect.

The first difficulty appears, when we define a Lie superalgebra $\mathfrak{g}_{\mathbb{Z}}$ over $\mathbb{Z}$. To define it, it is necessary to introduce the coroots appropriately. The second difficulty appears, when we reduce the proof to the rank one case. We will resolve these problems in $\S 2.5$ and $\S 4.3$ respectively. Finally we remark that the UCE of $\mathfrak{g}_{k}$ is $\overline{\mathfrak{g}}_{k}$, i.e., as opposed to the non-super case, the UCE of $\mathfrak{g}_{k}$ does not necessarily coincide with itself.

This paper is organized as follows: In Section 2, we will collect some fundamental concepts for basic classical Lie superalgebras, e.g. Dynkin diagrams, Cartan matrices and root systems. Further we will recall the classification theorem due to V.G.Kac and the realizations of these Lie superalgebras. Since the Killing form vanishes in some cases, in $\S 2.4$ we will describe an even supersymmetric invariant bilinear forms on basic classical Lie superalgebras. And in $\S 2.5$, we will define the coroots and study their properties. In Section 3, we will show the existence of a Chevalley type base (Theorem 3.9). In Section 4, will first summarize the definitions and basic properties of central extensions in $\S 4.1$. In $\S 4.2$, we state the main result of this paper (Theorem 4.7), and $\S 4.3$ is devoted to its proof. In the appendix, we will list some data for the basic classical Lie superalgebras, for the sake of readers convenience. 


\section{Acknowledgement}

We would like thank the referee for his careful reading and suggestions.

\section{Basic classical Lie superalgebras}

In this section, we recall the definition of basic classical Lie superalgebras and their fundamental properties. Through this section, we will work over the complex number field $\mathbb{C}$.

\subsection{Definitions}

We first list some notation for $\mathbb{Z}_{2}$-graded vector spaces. Let $\mathrm{V}=\mathrm{V}_{\overline{0}} \oplus \mathrm{V}_{\overline{1}}$ be a $\mathbb{Z}_{2}$-graded vector space. For $\sigma \in \mathbb{Z}_{2}$, we say that a vector $v \in \mathrm{V}_{\sigma}$ is homogeneous of degree $\sigma$ if $v \in \mathrm{V}_{\sigma}$. We set

$$
|v|:=\sigma .
$$

Let $\mathbb{C}^{m \mid n}$ be the $(m+n)$-dimensional $\mathbb{Z}_{2}$-graded vector space such that

$$
\left(\mathbb{C}^{m \mid n}\right)_{\overline{0}} \simeq \mathbb{C}^{m}, \quad\left(\mathbb{C}^{m \mid n}\right)_{\overline{1}} \simeq \mathbb{C}^{n} .
$$

We note that for $\mathbb{Z}_{2}$-graded vector spaces $\mathrm{V}$ and $\mathrm{W}, \operatorname{Hom}_{\mathbb{C}}(\mathrm{V}, \mathrm{W})$ is $\mathbb{Z}_{2}$-graded via

$$
\operatorname{Hom}_{\mathbb{C}}(\mathrm{V}, \mathrm{W})_{\tau}:=\bigoplus_{\sigma \in \mathbb{Z}_{2}} \operatorname{Hom}_{\mathbb{C}}\left(\mathrm{V}_{\sigma}, \mathrm{W}_{\sigma+\tau}\right), \quad \tau \in \mathbb{Z}_{2}
$$

Let $\mathfrak{g}:=\mathfrak{g}_{\overline{0}} \oplus \mathfrak{g}_{\overline{1}}$ be a Lie superalgebra over $\mathbb{C}$, i.e., there exists a bilinear map $[]:, \mathfrak{g} \times \mathfrak{g} \rightarrow \mathfrak{g}$ such that

1. $\left[\mathfrak{g}_{\sigma}, \mathfrak{g}_{\sigma^{\prime}}\right] \subset \mathfrak{g}_{\sigma+\sigma^{\prime}}$,

2. $[a, b]=-(-1)^{|a||b|}[b, a]$,

3. $[a,[b, c]]=[[a, b], c]+(-1)^{|a||b|}[b,[a, c]]$,

where $\sigma, \sigma^{\prime} \in \mathbb{Z}_{2}$ and $a, b, c \in \mathfrak{g}$ are homogeneous elements.

In this paper, we mainly deal with a class of Lie superalgebras called basic classical Lie superalgebras. To recall the definition, we introduce the following concepts:

Definition 2.1. For a bilinear form $\mathrm{F}: \mathfrak{g} \times \mathfrak{g} \rightarrow \mathbb{C}$, we say that

1. $\mathrm{F}$ is even if $\left(\mathfrak{g}_{\sigma}, \mathfrak{g}_{\sigma^{\prime}}\right)=0$ for $\sigma \neq \sigma^{\prime}$,

2. $\mathrm{F}$ is supersymmetric if $(a, b)=(-1)^{|a||b|}(b, a)$,

3. $\mathrm{F}$ is invariant if $([a, b], c)=(a,[b, c])$. 
We define a basic classical Lie superalgebra as follows [Kac2]:

Definiton 2.2. A finite dimensional Lie superalgebra $\mathfrak{g}$ is called $\mathbf{a}$ basic classical Lie superalgebra if

D1. $\mathfrak{g}$ is a simple Lie superalgebra i.e. has no non-trivial $\mathbb{Z}_{2}$-graded ideal,

D2. $\mathfrak{g}_{\overline{0}}$ is a reductive Lie algebra,

D3.there exists a non-degenerate even supersymmetric invariant bilinear form on

$\mathfrak{g}$.

The classification of the basic classical Lie superalgebras was carried out in [Kac1]. Let us recall the classification.

Theorem 2.3. The following list exhausts the basic classical Lie superalgebras over $\mathbb{C}$ :

1. simple Lie algebras,

2. simple Lie superalgebras of type

\begin{tabular}{|c|l|}
\hline $\mathrm{A}(m, n)$ & $m, n \geq 0$ and $m+n \geq 1$ \\
\hline $\mathrm{B}(m, n)$ & $m \geq 0$ and $n \geq 1$ \\
\hline $\mathrm{C}(n)$ & $n \geq 3$ \\
\hline $\mathrm{D}(m, n)$ & $m \geq 2$ and $n \geq 1$ \\
\hline $\mathrm{D}(2,1 ; a)$ & $a \neq 0,-1$ \\
\hline $\mathrm{F}(4)$ & \\
\hline $\mathrm{G}(3)$ & \\
\hline
\end{tabular}

Realizations of Lie superalgebras of type $\mathrm{A}(m, n), \mathrm{B}(m, n), \mathrm{C}(n)$ and $\mathrm{D}(m, n)$ will be described in the following subsections. Here we only make two remarks.

Remark 2.2.4. $\mathrm{A}(1,1) \simeq \mathrm{C}(2)$ and $\mathrm{D}(2,1 ; a) \simeq \mathrm{D}(2,1 ; b)$ if and only if $a$ and $b$ lie in the same orbit of the group generated by the transformations $a \mapsto-a-1$ and $a \mapsto 1 / a$, which is isomorphic to $\mathfrak{S}_{3}$.

Remark 2.2.5. $\mathfrak{g}$ is said to be classical if the conditions D1 and D2 hold [Kac1]. There are two series $\mathrm{P}(n)(n \geq 3)$ and $Q(n) \quad(n \geq 2)$ of classical Lie superalgebras, which are not basic classical. Note that $Q(n)$ has an odd supersymmetric invariant form.

\subsection{Examples}

In this subsection, we will explicitly realize the Lie superalgebras of type $\mathrm{A}(m, n)$, $\mathrm{B}(m, n), \mathrm{C}(n)$ and $\mathrm{D}(m, n)$ as subalgebras of the general linear Lie superalgebras. 
To do this, we first recall the general linear Lie superalgebra $\mathfrak{g l}(m, n)$. We put

$$
\mathfrak{g l}(m, n):=\operatorname{Hom}_{\mathbb{C}}\left(\mathbb{C}^{m \mid n}, \mathbb{C}^{m \mid n}\right)
$$

and regard it as a Lie superalgebra with the commutator

$$
[a, b]:=a b-(-1)^{|a||b|} b a .
$$

In the sequel, we fix a basis $\left\{e_{1}, \cdots, e_{m}, e_{m+1}, \cdots, e_{m+n}\right\}$ such that

$$
\left(\mathbb{C}^{m \mid n}\right)_{\overline{0}}=\bigoplus_{i=1}^{m} \mathbb{C} e_{i} \text { and }\left(\mathbb{C}^{m \mid n}\right)_{\overline{1}}=\bigoplus_{i=1}^{n} \mathbb{C} e_{m+i}
$$

For an element $a \in \mathfrak{g l}(m, n)$, let

$$
\left(\begin{array}{ll}
\mathrm{A} & \mathrm{B} \\
\mathrm{C} & \mathrm{D}
\end{array}\right)
$$

be the matrix representation of $a$ with respect to the above basis. We define the supertrace of $a$ by

$$
\operatorname{str} a:=\operatorname{tr} \mathrm{A}-\operatorname{tr} \mathrm{D} .
$$

Moreover we set

$$
\mathfrak{s l}(m, n):=\{a \in \mathfrak{g l}(m, n) \mid \operatorname{str} a=0\}
$$

and call it a special linear Lie superalgebra. Notice that the identity matrix $\mathrm{I}_{2 n}$ is an element of $\mathfrak{s l}(n, n)$, moreover $\mathbb{C I}_{2 n}$ is the center of $\mathfrak{s l}(n, n)$.

Example 2.6. The Lie superalgebra of type $\mathrm{A}(m, n)$ is defined by

$$
\mathrm{A}(m, n):= \begin{cases}\mathfrak{s l}(m+1, n+1) & \text { if } m \neq n \\ \mathfrak{s l}(m+1, n+1) / \mathbb{C I}_{2 n+2} & \text { if } m=n\end{cases}
$$

Next we recall the Lie superalgebras of type $\mathrm{B}(m, n), \mathrm{C}(n)$ and $\mathrm{D}(m, n)$. For this purpose, we introduce the ortho-symplectic Lie superalgebra osp $(m, n)$. We set 


$$
\begin{aligned}
& \mathfrak{o s p}(2 m+1,2 n):=
\end{aligned}
$$

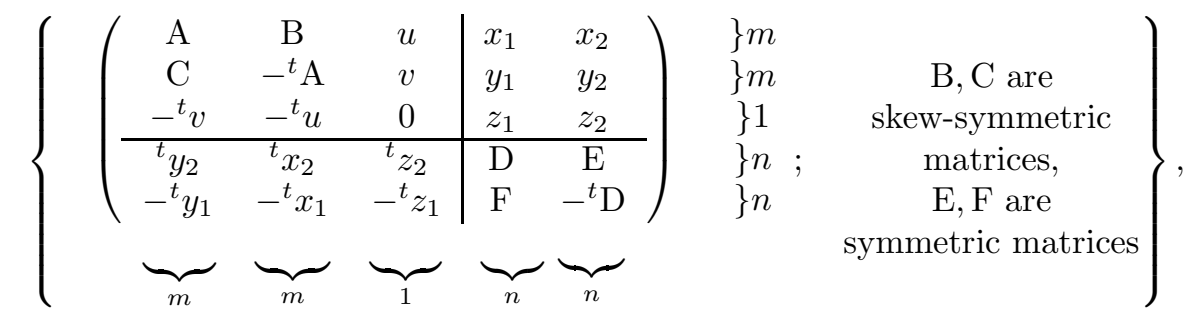

$\mathfrak{o s p}(2 m, 2 n):=$

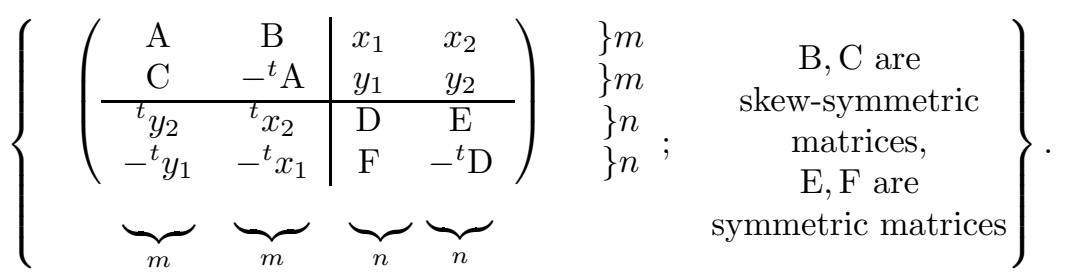

Example 2.7. The Lie superalgebras of type $\mathrm{B}(m, n), \mathrm{C}(n)$ and $\mathrm{D}(m, n)$ are defined by

$$
\begin{aligned}
\mathrm{B}(m, n) & :=\mathfrak{o s p}(2 m+1,2 n) & & (m \geq 0, n \geq 1) \\
\mathrm{D}(m, n) & :=\mathfrak{o s p}(2 m, 2 n) & & (m \geq 2, n \geq 1) \\
\mathrm{C}(n) & :=\mathfrak{o s p}(2,2 n-2) & & (n \geq 3) .
\end{aligned}
$$

\subsection{Contragradient Lie superalgebras}

In this subsection, we will define all basic classical Lie superalgebras as contragradient Lie superalgebras.

We start with the definition of contragradient Lie superalgebras. The contragradient Lie superalgebras are defined from the following data:

Let $\mathrm{I}$ be the index set $\{1,2, \cdots, n\}$, and $\tau$ a subset of $\mathrm{I}$. Let $\mathrm{A}=\left(a_{i, j}\right)_{i, j \in \mathrm{I}}$ be a complex $n \times n$ matrix of rank $l$. Let $\tilde{\mathfrak{h}}$ be a $(2 n-l)$-dimensional $\mathbb{C}$-vector space and $\tilde{Q}$ the free abelian group of rank $n$ with basis $\tilde{\Pi}:=\left\{\tilde{\alpha}_{i} \mid i \in \mathrm{I}\right\} \subset \tilde{\mathfrak{h}}^{*}$. We can choose a subset $\left\{\tilde{h}_{i} \mid i \in \mathrm{I}\right\}$ of $\tilde{\mathfrak{h}}$ such that

1. $\left\{\tilde{h}_{i} \mid i \in \mathrm{I}\right\}$ is linearly independent,

2. $\tilde{\alpha}_{i}\left(\tilde{h}_{j}\right)=a_{j, i}$ for any $i, j \in \mathrm{I}$.

Using this data, we define the contragradient Lie superalgebra. 
Definition 2.8. The contragradient Lie superalgebra $\tilde{\mathfrak{g}}$ is the Lie superalgebra which satisfies the following conditions:

$$
\text { C1. } \tilde{\mathfrak{g}}=\bigoplus_{\tilde{\alpha} \in \tilde{Q}} \tilde{\mathfrak{g}}_{\tilde{\alpha}}: \tilde{Q}_{\text {-graded }}
$$

C2. There exist generators $\left\{\tilde{e}_{i}, \tilde{f}_{i}, \tilde{h} \mid i \in \mathrm{I}, h \in \tilde{\mathfrak{h}}\right\}$ such that

$$
\tilde{\mathfrak{g}}_{0}=\tilde{\mathfrak{h}}, \quad \tilde{\mathfrak{g}}_{\tilde{\alpha}_{i}}=\mathbb{C} \tilde{e}_{i}, \quad \tilde{\mathfrak{g}}_{-\tilde{\alpha}_{i}}=\mathbb{C} \tilde{f}_{i}, \quad|\tilde{h}|:=\overline{0}, \quad\left|\tilde{e}_{i}\right|=\left|\tilde{f}_{i}\right|=\left\{\begin{array}{ll}
\overline{1} & \text { if } i \in \tau, \\
\overline{0} & \text { if } i \notin \tau,
\end{array},\right.
$$

and satisfy commutation relations

$$
\begin{aligned}
& {\left[\tilde{h}, \tilde{h}^{\prime}\right]=0\left(\tilde{h}, \tilde{h}^{\prime} \in \tilde{\mathfrak{h}}\right),\left[\tilde{e}_{i}, \tilde{f}_{j}\right]=\delta_{i, j} \tilde{h}_{i},} \\
& {\left[\tilde{h}, \tilde{e}_{i}\right]=\tilde{\alpha}_{i}(\tilde{h}) \tilde{e}_{i},\left[\tilde{h}, \tilde{f}_{i}\right]=-\tilde{\alpha}_{i}(\tilde{h}) \tilde{f}_{i} .}
\end{aligned}
$$

C3.Every $\tilde{Q}$-graded ideal $\mathfrak{r}$ of $\tilde{\mathfrak{g}}$ such that $\mathfrak{r} \cap \tilde{\mathfrak{h}}=\{0\}$ is zero. and

The matrix $\mathrm{A}$ is called the Cartan matrix of $\tilde{\mathfrak{g}}$. We set $\tilde{Q}^{+}:=\sum_{i \in \mathrm{I}} \mathbb{Z}_{\geq 0} \tilde{\alpha}_{i}$

$$
\begin{aligned}
& \tilde{\Delta}:=\left\{\tilde{\alpha} \in \tilde{Q} \backslash\{0\} \mid \tilde{\mathfrak{g}}_{\tilde{\alpha}} \neq\{0\}\right\}, \\
& \tilde{\Delta}^{+}:=\left\{\tilde{\alpha} \in \tilde{Q}^{+} \backslash\{0\} \mid \tilde{\mathfrak{g}}_{\tilde{\alpha}} \neq\{0\}\right\}, \\
& \tilde{\Delta}_{\sigma}^{+}:=\left\{\tilde{\alpha} \in \tilde{Q}^{+} \backslash\{0\} \mid \tilde{\mathfrak{g}}_{\sigma} \cap \tilde{\mathfrak{g}}_{\tilde{\alpha}} \neq\{0\}\right\} .
\end{aligned}
$$

It is easy to see that $\tilde{\mathfrak{g}}$ has a triangular decomposition

$$
\tilde{\mathfrak{g}}=\tilde{\mathfrak{n}}^{+} \oplus \tilde{\mathfrak{h}} \oplus \tilde{\mathfrak{n}}^{-} \quad\left(\tilde{\mathfrak{n}}^{ \pm}:=\bigoplus_{ \pm \tilde{\alpha} \in \tilde{\Delta}^{+}} \tilde{\mathfrak{g}}_{\tilde{\alpha}}\right),
$$

and hence $\tilde{\Delta}=\tilde{\Delta}^{+} \sqcup\left(-\tilde{\Delta}^{+}\right)$. Furthermore, by definition, we have $\tilde{\Delta}^{+}=\tilde{\Delta}_{\overline{0}}^{+} \sqcup$ $\tilde{\Delta}_{\overline{1}}^{+}$(disjoint union).

On the simplicity of a contragradient Lie superalgebra, we have the following proposition [Kac1]

Proposition 2.9. Let $\tilde{\mathfrak{g}}$ be a contragradient Lie superalgebra with the Cartan matrix $\mathrm{A}=\left(a_{i, j}\right)$ and let $\tilde{\mathfrak{g}}^{\prime}$ be the derived subalgebra $[\tilde{\mathfrak{g}}, \tilde{\mathfrak{g}}]$ of $\tilde{\mathfrak{g}}$. We denote the center of $\tilde{\mathfrak{g}}$ by $\mathrm{C}$. Then we have

1. $\mathrm{C}=\left\{\sum_{i \in \mathrm{I}} c_{i} h_{i} \mid c_{i} \in \mathbb{C}\right.$ such that $\left.\sum_{i \in \mathrm{I}} a_{i, j} c_{i}=0\left({ }^{\forall} j \in \mathrm{I}\right)\right\} \subset \tilde{\mathfrak{g}}^{\prime}$.

2. $\tilde{\mathfrak{g}}^{\prime} / \mathrm{C}$ is graded simple in the $\tilde{Q}$-grading if and only if $\mathrm{A}$ is indecomposable.

The following theorem due to V.G. Kac tells us which Cartan matrix corresponds to a basic classical Lie superalgebra.

Theorem 2.10. [[Kac1]] The Lie superalgebra $\mathfrak{g}:=\tilde{\mathfrak{g}}^{\prime} / \mathrm{C}$ with a Cartan matrix whose Dynkin diagram is one of those in Appendix $A$ is a basic classical Lie superalgebra. 
Corollary 2.11. Let A be a Cartan matrix of a basic classical Lie superalgebra. Then we have

$$
\mathrm{C}=\left\{\begin{array}{ll}
\mathbb{C}\left[\sum_{i=1}^{n} i\left(\tilde{h}_{i}-\tilde{h}_{2 n+2-i}\right)-(n+1) \tilde{h}_{n+1}\right] & \begin{array}{l}
\text { if } \mathrm{A} \text { is of type } \mathrm{A}(n, n)\left({ }^{\exists} n\right) \\
\{0\}
\end{array} \\
\text { otherwise }
\end{array} .\right.
$$

In the sequel, we will need the following notation. We denote the canonical projection $\tilde{\mathfrak{g}}^{\prime} \rightarrow \mathfrak{g}$ by $\phi$. We set $\mathfrak{h}:=\phi\left(\tilde{\mathfrak{g}}^{\prime} \cap \tilde{\mathfrak{h}}\right)$ and call it a Cartan subalgebra of $\mathfrak{g}$. Let us denote the image of $\tilde{x} \in \tilde{\mathfrak{g}}^{\prime}$ under the map $\phi$ by $x$. The dual of the map $\left.\phi\right|_{\tilde{\mathfrak{h}} \cap \tilde{\mathfrak{a}}^{\prime}}$ defines an inclusion $\iota: \mathfrak{h}^{*} \hookrightarrow\left(\tilde{\mathfrak{h}} \cap \tilde{\mathfrak{g}}^{\prime}\right)^{*}$. Similarly, we define the map $\psi: \tilde{\mathfrak{h}}^{*} \rightarrow\left(\tilde{\mathfrak{h}} \cap \tilde{\mathfrak{g}}^{\prime}\right)^{*}$ as the dual of the natural inclusion $\tilde{\mathfrak{h}} \cap \tilde{\mathfrak{g}}^{\prime} \hookrightarrow \tilde{\mathfrak{h}}$. Since $\left.\tilde{\alpha}\right|_{\mathrm{C}} \equiv 0$ for any $\tilde{\alpha} \in \tilde{Q}$, we can regard $\psi(\tilde{Q}) \subset$ I $m \iota$. Therefore, for $\tilde{\alpha} \in \tilde{Q}$, we define $\alpha \in \mathfrak{h}$ by $\alpha:=\left(\iota^{-1} \circ \psi\right)(\tilde{\alpha})$ and set $Q:=\left(\iota^{-1} \circ \psi\right)(\tilde{Q})$. We also define

$$
\Delta:=\left(\iota^{-1} \circ \psi\right)(\tilde{\Delta}), \quad \Delta^{+}:=\left(\iota^{-1} \circ \psi\right)\left(\tilde{\Delta}^{+}\right), \quad \Delta_{\sigma}^{+}:=\left(\iota^{-1} \circ \psi\right)\left(\tilde{\Delta}_{\sigma}^{+}\right) .
$$

We call $\alpha_{i}:=\left(\iota^{-1} \circ \psi\right)\left(\tilde{\alpha}_{i}\right)$ (resp. $\left.h_{i}:=\phi\left(\tilde{h}_{i}\right)\right)(i \in \mathrm{I})$ the $i$-th simple root (resp. simple coroot), and set $\Pi:=\left\{\alpha_{i} \mid i \in \mathrm{I}\right\}$. For $\tilde{\alpha} \in \tilde{Q}$, we also set $\mathfrak{g}_{\alpha}:=\phi\left(\tilde{\mathfrak{g}}_{\tilde{\alpha}}\right)$.

Here we notice that, from Corollary 2.11, in the case of $\mathrm{A}(n, n)$, we have

$$
\sum_{i=1}^{n} i\left(\alpha_{i}+\alpha_{2 n+2-i}\right)+(n+1) \alpha_{n+1}=0 .
$$

Let us recall some properties of the root systems of the basic classical Lie superalgebras. The following proposition is known:

Proposition 2.12. [[Kac1]] If $\mathfrak{g}$ is a basic classical Lie superalgebra which is not of type $\mathrm{A}(1,1)$, then $\operatorname{dim} \mathfrak{g}_{\alpha}=1$ for any $\alpha \in \Delta$.

The triangular decomposition of $\tilde{\mathfrak{g}}$ yields

$$
\mathfrak{g}=\mathfrak{n}^{+} \oplus \mathfrak{h} \oplus \mathfrak{n}^{-} \quad\left(\mathfrak{n}^{ \pm}:=\bigoplus_{ \pm \alpha \in \Delta^{+}} \mathfrak{g}_{\alpha}\right),
$$

and hence $\Delta=\Delta^{+} \sqcup\left(-\Delta^{+}\right)$. Furthermore, $\Delta^{+}=\Delta_{\overline{0}}^{+} \sqcup \Delta_{\overline{1}}^{+}$holds. Hence, in the sequel, we denote the parity of $\mathfrak{g}_{\alpha}$ by $|\alpha|$.

\section{Remark 2.2.13.}

1. For the basic classical Lie superalgebra $\mathfrak{g}$ of type $\mathrm{A}(1,1)$, the relations $\alpha_{1}+$ $2 \alpha_{2}+\alpha_{3}=0$, and hence $\alpha_{1}+\alpha_{2}=-\alpha_{2}-\alpha_{3}, \alpha_{1}+\alpha_{2}+\alpha_{3}=-\alpha_{2}$ hold. These roots are distinguished in the corresponding contragradient Lie superalgebra $\tilde{\mathfrak{g}}$. Namely, we have $\operatorname{dim} \tilde{\mathfrak{g}}_{\tilde{\alpha}}=1$ for any $\tilde{\alpha} \in \tilde{\Delta}$.

2. In the case of $\mathrm{A}(n, n)$ for $n \in 2 \mathbb{Z}_{>0}, Q \subset \mathfrak{h}^{*}$ itself is not $\mathbb{Z}_{2}$-graded. Nevertheless, we can define the $\mathbb{Z}_{2}$-gradation on $\Delta$. 


\subsection{Non-degenerate even supersymmetric bilinear form}

In this subsection, we will describe even supersymmetric invariant bilinear forms on the basic classical Lie superalgebras explicitly.

Let $\mathfrak{g}$ be one of $\mathfrak{s l}(m, n)$ and $\mathfrak{o s p}(m, n)$. We define $(\cdot, \cdot): \mathfrak{g} \times \mathfrak{g} \rightarrow \mathbb{C}$ by

$$
(a, b):=\operatorname{str}(a b) \quad(a, b \in \mathfrak{g}),
$$

where we regard $\mathfrak{g}$ as a subalgebra of $\mathfrak{g l}(m, n)$. By definition, we immediately see that

Proposition 2.14. $(\cdot, \cdot)$ is an even supersymmetric invariant bilinear form on $\mathfrak{g}$.

As a corollary, we have

Corollary 2.15. The radical of the form $(\cdot, \cdot)$ is a $\mathbb{Z}_{2}$-graded ideal of $\mathfrak{g}$.

Therefore, in addition if $\mathfrak{g}$ is a simple Lie superalgebra, then $\operatorname{rad}(\cdot, \cdot)=\{0\}$. Notice that, in the case where $\mathfrak{g}=\mathfrak{s l}(n+1, n+1), \mathfrak{g}$ is not simple. But in this case we have

Lemma 2.16. Suppose that $\mathfrak{g}=\mathfrak{s l}(n+1, n+1)$. Then we have $\mathbb{C I}_{2 n+2} \subset \operatorname{rad}(\cdot, \cdot)$, and hence $(\cdot, \cdot)$ defines an even supersymmetric bilinear form on $\mathrm{A}(n, n):=\mathfrak{s l}(n+$ $1, n+1) / \mathbb{C I}_{2 n+2}$.

Accordingly we have

Proposition 2.17. Let $\mathfrak{g}$ be a basic classical Lie superalgebra of type $\mathrm{A}(m, n)$, $\mathrm{B}(m, n), \mathrm{C}(m, n)$ or $\mathrm{D}(m, n)$. The pairing $(\cdot, \cdot): \mathfrak{g} \times \mathfrak{g} \rightarrow \mathbb{C}$ defined by $(a, b):=$ $\operatorname{str}(a b)$ gives a non-degenerate even supersymmetric invariant bilinear form on $\mathfrak{g}$.

Proof. Since $\mathfrak{g}$ is simple, it follows from Corollary 2.15 that $\operatorname{rad}(\cdot, \cdot)=\{0\}$. Hence $(\cdot, \cdot)$ is non-degenerate.

Hence the supertrace gives a non-degenerate even supersymmetric bilinear form on $\mathfrak{g}$, if $\mathfrak{g}$ is of type $\mathrm{A}(m, n), \mathrm{B}(m, n), \mathrm{C}(m, n)$ or $\mathrm{D}(m, n)$. Generically, the Killing form defined below gives such a form on $\mathfrak{g}$.

Definition 2.18. Let $\mathfrak{g}$ be a finite dimensional Lie superalgebra over $\mathbb{C}$. The bilinear form $\mathrm{F}: \mathfrak{g} \times \mathfrak{g} \rightarrow \mathbb{C}$ such that $\mathrm{F}(a, b):=\operatorname{str}(\operatorname{ad}(a) \circ \operatorname{ad}(b))$ is called the Killing form on $\mathfrak{g}$.

By definition, the Killing form is an even supersymmetric invariant bilinear form on $\mathfrak{g}$. In the case where $\mathfrak{g}$ is a basic classical Lie superalgebra, the Killing 
form is non-degenerate if $\mathfrak{g}$ is not of type $\mathrm{A}(n, n), \mathrm{D}(n+1, n)$ and $\mathrm{D}(2,1 ; a)$. Hence if $\mathfrak{g}$ is of type $\mathrm{F}(4)$ or $\mathrm{G}(3)$, then the Killing form gives an even supersymmetric bilinear form.

Since non-degenerate even supersymmetric bilinear forms on $\mathrm{A}(n, n)$ and $\mathrm{D}(n+$ $1, n)$ are given by the supertrace as above, it is enough to describe it for $\mathrm{D}(2,1 ; a)$.

Here let us discuss an even supersymmetric bilinear form on $\mathrm{D}(2,1 ; a)$. At the same time, we consider the case of $\mathrm{D}(2,1 ;-1)$, which is not a simple Lie superalgebra, but $\mathrm{D}(2,1 ;-1)$ gives the universal central extension of $\mathrm{A}(1,1)$. To give an explicit even supersymmetric invariant bilinear form $(\cdot, \cdot)$ on $\mathrm{D}(2,1 ; a)$, we fix its root vectors. For the Cartan matrix and the root system of $\mathrm{D}(2,1 ; a)$, see Appendix A. From now on, let us assume that $a \neq 0$. We set

$$
\begin{aligned}
& X_{\alpha_{1}}:=e_{1}, \quad X_{\alpha_{2}}:=e_{2}, \quad X_{\alpha_{3}}:=e_{3}, \\
& X_{-\alpha_{1}}:=f_{1}, \quad X_{-\alpha_{2}}:=f_{2}, \quad X_{-\alpha_{3}}:=f_{3}, \\
& X_{\alpha_{1}+\alpha_{2}}:=\left[X_{\alpha_{1}}, X_{\alpha_{2}}\right], \quad X_{-\alpha_{1}-\alpha_{2}}:=\left[X_{-\alpha_{2}}, X_{-\alpha_{1}}\right], \\
& X_{\alpha_{2}+\alpha_{3}}:=\left[X_{\alpha_{3}}, X_{\alpha_{2}}\right], \quad X_{-\alpha_{2}-\alpha_{3}}:=\left[X_{-\alpha_{2}}, X_{-\alpha_{3}}\right], \\
& X_{\alpha_{1}+\alpha_{2}+\alpha_{3}}:=\left[X_{\alpha_{1}}, X_{\alpha_{2}+\alpha_{3}}\right], \quad X_{-\alpha_{1}-\alpha_{2}-\alpha_{3}}:=\left[X_{-\alpha_{2}-\alpha_{3}}, X_{-\alpha_{1}}\right], \\
& X_{ \pm\left(\alpha_{1}+2 \alpha_{2}+\alpha_{3}\right)}:=\left\{\begin{array}{lll} 
\pm \frac{1}{a+1}\left[X_{ \pm\left(\alpha_{1}+\alpha_{2}+\alpha_{3}\right)}, X_{ \pm \alpha_{2}}\right] & \text { if } & a \neq-1 \\
\pm\left[X_{ \pm\left(\alpha_{1}+\alpha_{2}+\alpha_{3}\right)}, X_{ \pm \alpha_{2}}\right] & \text { if } & a=-1
\end{array}\right.
\end{aligned}
$$

The bilinear form $(\cdot, \cdot)$ on $\mathrm{D}(2,1 ; a)$ is explicitly given by

$$
\begin{aligned}
& \left(h_{1}, h_{1}\right)=2, \quad\left(h_{1}, h_{2}\right)=1, \quad\left(h_{1}, h_{3}\right)=0, \\
& \left(h_{2}, h_{2}\right)=0, \quad\left(h_{2}, h_{3}\right)=1, \quad\left(h_{3}, h_{3}\right)=\frac{2}{a}, \\
& \left(X_{\alpha_{1}}, X_{-\alpha_{1}}\right)=1, \quad\left(X_{\alpha_{2}}, X_{-\alpha_{2}}\right)=-1, \quad\left(X_{\alpha_{3}}, X_{-\alpha_{3}}\right)=\frac{1}{a}, \\
& \left(X_{\alpha_{1}+\alpha_{2}}, X_{-\alpha_{1}-\alpha_{2}}\right)=-1, \quad\left(X_{\alpha_{2}+\alpha_{3}}, X_{-\alpha_{2}-\alpha_{3}}\right)=-1, \\
& \left(X_{\alpha_{1}+\alpha_{2}+\alpha_{3}}, X_{-\alpha_{1}-\alpha_{2}-\alpha_{3}}\right)=-1, \\
& \left(X_{\alpha_{1}+2 \alpha_{2}+\alpha_{3}}, X_{-\alpha_{1}-2 \alpha_{2}-\alpha_{3}}\right)=\left\{\begin{array}{lll}
-\frac{1}{a+1} & \text { if } & a \neq-1 \\
0 & \text { if } & a=-1
\end{array} .\right.
\end{aligned}
$$

Notice that if $a \neq-1$, then $(\cdot, \cdot)$ is non-degenerate on $\mathrm{D}(2,1 ; a)$.

For later use, we introduce Lie superalgebras $\mathfrak{d}$ and $\overline{\mathfrak{g}}$. Let $\mathfrak{d}$ be the Lie superalgebra of type $\mathrm{D}(2,1 ;-1)$. From the commutation relations of $\mathfrak{d}$, it is easy to see that the vectors $X_{ \pm\left(\alpha_{1}+2 \alpha_{2}+\alpha_{3}\right)}$ are central elements of $\mathfrak{d}$ and further

$$
0 \longrightarrow \bigoplus_{\mu= \pm 1} \mathbb{C} X_{\mu\left(\alpha_{1}+2 \alpha_{2}+\alpha_{3}\right)} \longrightarrow \mathfrak{d} \longrightarrow \mathfrak{s l}(2,2) \longrightarrow 0
$$

is exact. Moreover, for a basic classical Lie superalgebra $\mathfrak{g}$, we introduce the Lie superalgebra $\overline{\mathfrak{g}}$ by

$$
\overline{\mathfrak{g}}:=\left\{\begin{array}{ll}
\mathfrak{g} & \text { if } \mathfrak{g} \text { is not of type } \mathrm{A}(n, n)\left({ }^{\forall} n\right) \\
\mathfrak{d} & \text { if } \mathfrak{g} \text { is of type A }(1,1) \\
\mathfrak{s l}(n+1, n+1) & \text { if } \mathfrak{g} \text { is of type A }(n, n)\left({ }^{\exists} n>1\right)
\end{array},\right.
$$

and denote the canonical projection $\overline{\mathfrak{g}} \longrightarrow \mathfrak{g}$ by $\pi$. Then we have an exact 
sequence

$$
0 \longrightarrow \mathbb{C}^{\oplus m} \longrightarrow \overline{\mathfrak{g}} \longrightarrow \mathfrak{g} \longrightarrow 0
$$

where

$$
m:=\left\{\begin{array}{ll}
0 & \text { if } \mathfrak{g} \text { is not of type } \mathrm{A}(n, n)\left({ }^{\forall} n\right) \\
3 & \text { if } \mathfrak{g} \text { is of type } \mathrm{A}(1,1) \\
1 & \text { if } \mathfrak{g} \text { is of type } \mathrm{A}(n, n)(n \geq 2)
\end{array} .\right.
$$

By abuse of notation, we will sometimes use the same symbols to denote root vectors of $\mathfrak{g}$ and $\overline{\mathfrak{g}}$.

Remark 2.2.19. As was seen in this subsection, $\overline{\mathfrak{g}}$ has an even supersymmetric invariant bilinear form $(\cdot, \cdot)$, and further by Corollary 2.15 and (3), we have $\operatorname{Rad}(\cdot, \cdot)=\operatorname{ker} \pi$.

\subsection{Coroots}

In this subsection, we will define the coroot $H_{\alpha}$ for each $\alpha \in \Delta$, and study their property.

Let $\mathrm{A}:=\left(a_{i, j}\right)_{i, j \in \mathrm{I}}$ be the Cartan matrix of a basic classical Lie superalgebra $\mathfrak{g}$ and let $\mathrm{D}:=\operatorname{diag}\left(\varepsilon_{i}\right)_{i \in \mathrm{I}}$ and $\mathrm{B}:=\left(b_{i, j}\right)_{i, j \in \mathrm{I}}$ be diagonal and symmetric matrices such that $\mathrm{A}=\mathrm{DB}$. We see that

$$
\varepsilon_{j} a_{i, j}=\varepsilon_{i} a_{j, i} .
$$

In the sequel, we will fix the Dynkin diagrams as in Appendix A. Hence an odd simple root is unique, and is denoted by $\alpha_{i_{0}}$. We set

$$
\overline{\mathrm{W}}:=\left\langle r_{i} \mid i \in \mathrm{I} \backslash\left\{i_{0}\right\}\right\rangle,
$$

where $r_{i}$ denotes the $i$-th simple reflection. Note that $\overline{\mathrm{W}}$ is a subgroup of the Weyl group $W$ of $\mathfrak{g}$, i.e., the Weyl group of $\mathfrak{g}_{\overline{0}}$.

For each $\alpha \in \Delta$, let us define

$$
\varepsilon_{\alpha}:=\left\{\begin{array}{cc}
\frac{2}{(\alpha, \alpha)} & \text { if } \quad(\alpha, \alpha) \neq 0 \\
\varepsilon_{i_{0}} & \text { if }(\alpha, \alpha)=0
\end{array} .\right.
$$

By the Weyl group invariance of $(\cdot, \cdot)$, we have

$$
\varepsilon_{w \alpha}=\varepsilon_{\alpha}, \quad{ }^{\forall} \alpha \in \Delta, \quad{ }^{\forall} \alpha \in \mathrm{W} .
$$

Now we define the coroots as follows:

Definition 2.20. For $\alpha=\sum_{i \in \mathrm{I}} k_{i} \alpha_{i} \in \Delta$, we set

$$
H_{\alpha}:=\varepsilon_{\alpha} \sum_{i \in \mathrm{I}} k_{i} \varepsilon_{i}^{-1} h_{i}
$$


where $h_{i}$ is the $i$-th simple coroot. We call $H_{\alpha}$ the coroot associated with a root $\alpha$.

By definition, we have

$$
H_{-\alpha}=-H_{\alpha}
$$

and $H_{\alpha_{i}}=h_{i}$. Moreover, the following proposition holds:

Proposition 2.21. For any $\alpha \in \Delta$ and $w \in \mathrm{W}$, we have

$$
w H_{\alpha}=H_{w \alpha} \text {. }
$$

Proof. Suppose that $\alpha_{j} \in \Delta^{+} \cap \Pi$ and set $\alpha:=\sum_{i} k_{i} \alpha_{i}$. Then, one has

$$
\begin{aligned}
r_{j}(\alpha) & =\sum k_{i}\left(\alpha_{i}-a_{j, i} \alpha_{j}\right) \\
& =\sum_{i \neq j} k_{i} \alpha_{i}-\left(k_{j}+\sum_{i \neq j} k_{i} a_{j, i}\right) \alpha_{j} .
\end{aligned}
$$

On the other hand, it follows from (6), (7), and the definition of $H_{\alpha}$ (8) that

$$
\begin{aligned}
r_{j} H_{\alpha} & =\varepsilon_{\alpha} \sum_{i} k_{i} \varepsilon_{i}^{-1}\left(H_{\alpha_{i}}-a_{i, j} H_{\alpha_{j}}\right) \\
& =\varepsilon_{r_{j} \alpha}\left\{\sum_{i \neq j} k_{i} \varepsilon_{i}^{-1} H_{\alpha_{i}}-\varepsilon_{j}^{-1}\left(k_{j}+\sum_{i \neq j} k_{i} a_{j, i}\right) H_{\alpha_{j}}\right\} .
\end{aligned}
$$

Thus we have $r_{j} H_{\alpha}=H_{r_{j} \alpha}$ and the proposition is proved for $w \in \overline{\mathrm{W}}$. Next let us prove the proposition for a generator say $r_{\beta}$ of $\mathrm{W}$ that is not an element of $\overline{\mathrm{W}}$. In particular, we can choose the root $\beta$ as follows:

\begin{tabular}{|c|l|}
\hline type of $\mathfrak{g}$ & $\beta$ \\
\hline $\mathrm{B}(m, n)$ & $2 \delta_{n}=2 \alpha_{n}+2 \alpha_{n+1}+\cdots+2 \alpha_{m+n}$ \\
\hline $\mathrm{D}(m, n)$ & $2 \delta_{n}=2 \alpha_{n}+2 \alpha_{n+1}+\cdots+2 \alpha_{m+n-2}+\alpha_{m+n-1}+\alpha_{m+n}$ \\
\hline $\mathrm{D}(2,1: a)$ & $2 \epsilon_{2}=\alpha_{1}+2 \alpha_{2}+\alpha_{3}$ \\
\hline $\mathrm{F}(4)$ & $\delta_{1}=2 \alpha_{1}+3 \alpha_{2}+2 \alpha_{3}+\alpha_{4}$ \\
\hline $\mathrm{G}(3)$ & $2 \delta_{1}=2 \alpha_{1}+4 \alpha_{2}+2 \alpha_{3}$ \\
\hline
\end{tabular}

We remark that the cases $\mathrm{A}(m, n)$ and $\mathrm{C}(n)$ are excluded, since the group $\mathrm{W}$ coincides with the group $\overline{\mathrm{W}}$. From the above computation and (7), we have only to check

$$
\varepsilon_{\beta} \beta\left(H_{\alpha_{i}}\right)=\varepsilon_{i} \alpha_{i}\left(H_{\beta}\right) .
$$

This can be done through case-by-case calculation. 
Lemma 2.22. For any $\gamma \in \Delta^{+}$such that $(\gamma, \gamma)=0$, we have $\gamma \in \overline{\mathrm{W}} \alpha_{i_{0}}$.

Proof. Suppose that $\gamma=\sum_{i \in \mathrm{I}} k_{i} \alpha_{i} \in \Delta^{+}$such that $(\gamma, \gamma)=0$. We set ht $\gamma:=$ $\sum_{i \in \mathrm{I}} k_{i}$. We prove the lemma by induction on ht $\gamma$. If ht $\gamma=1$, then $\gamma=\alpha_{i_{0}}$. Hence we may assume that ht $\gamma>1$. Since

$$
r_{s}(\gamma)=\gamma-\frac{2\left(\gamma, \alpha_{s}\right)}{\left(\alpha_{s}, \alpha_{s}\right)} \alpha_{s}
$$

it suffices to show that there exists $s \in \mathrm{I} \backslash\left\{i_{0}\right\}$ such that $\operatorname{ht} \gamma>\operatorname{ht}\left(r_{s} \gamma\right)$, i.e.,

$$
\frac{2\left(\gamma, \alpha_{s}\right)}{\left(\alpha_{s}, \alpha_{s}\right)}>0
$$

Here let us take $\epsilon_{i}, \delta_{j}$ as in Appendix A. For each $\gamma \in \Delta^{+}$, we may choose the

\begin{tabular}{|c|c|c|}
\hline type of $\mathfrak{g}$ & $\gamma \in \Delta$ s.t. $(\gamma, \gamma)=0$ & simple root $\alpha_{s}$ \\
\hline $\mathrm{A}(m, n)$ & $\epsilon_{i}-\delta_{j}((i, j) \neq(m+1,1))$ & $\begin{array}{ll}\epsilon_{i}-\epsilon_{i+1} & (i \neq m+1) \\
\delta_{j-1}-\delta_{j} & (j \neq 1)\end{array}$ \\
\hline \multirow{2}{*}{$\begin{array}{l}\mathrm{B}(m, n) \\
(m \neq 0)\end{array}$} & $\delta_{i}+\epsilon_{j}$ & $\begin{array}{ll}\epsilon_{j}-\epsilon_{j+1} & (j \neq m) \\
\epsilon_{m} & (j=m)\end{array}$ \\
\hline & $\delta_{i}-\epsilon_{j}((i, j) \neq(n, 1))$ & $\begin{array}{ll}\delta_{i}-\delta_{i+1} & (i \neq n) \\
\epsilon_{j-1}-\epsilon_{j} & (j \neq 1)\end{array}$ \\
\hline \multirow[t]{2}{*}{$\mathrm{C}(n)$} & $\epsilon_{1}+\delta_{i}$ & $\begin{array}{ll}\delta_{i}-\delta_{i+1} & (i \neq n-1) \\
2 \delta_{n-1} & (i=n-1)\end{array}$ \\
\hline & $\epsilon_{1}-\delta_{i}(i \neq 1)$ & $\delta_{i-1}-\delta_{i}(i \neq 1)$ \\
\hline \multirow[t]{2}{*}{$\mathrm{D}(m, n)$} & $\delta_{i}+\epsilon_{j}$ & $\begin{array}{ll}\epsilon_{j}-\epsilon_{j+1} & (j \neq m) \\
\epsilon_{m-1}+\epsilon_{m} & (j=m)\end{array}$ \\
\hline & $\delta_{i}-\epsilon_{j}((i, j) \neq(n, 1))$ & $\begin{array}{ll}\delta_{i}-\delta_{i+1} & (i \neq n) \\
\epsilon_{j-1}-\epsilon_{j} & (j \neq 1)\end{array}$ \\
\hline \multirow[t]{2}{*}{$\overline{\mathrm{D}(2,1 ; a)}$} & $\epsilon_{2}+\epsilon_{1} \pm \epsilon_{3}$ & $2 \epsilon_{1}$ \\
\hline & $\epsilon_{2}-\epsilon_{1}+\epsilon_{3}$ & $2 \epsilon_{3}$ \\
\hline \multirow[t]{3}{*}{$\mathrm{F}(4)$} & $\frac{1}{2}\left(\delta_{1}-\epsilon_{1} \pm \epsilon_{2} \pm \epsilon_{3}\right)$ & $-\epsilon_{1}$ \\
\hline & $\frac{1}{2}\left(\delta_{1}+\epsilon_{1}-\epsilon_{2} \pm \epsilon_{3}\right)$ & $\epsilon_{1}-\epsilon_{2}$ \\
\hline & $\frac{1}{2}\left(\delta_{1}+\epsilon_{1}+\epsilon_{2}-\epsilon_{3}\right)$ & $\epsilon_{2}-\epsilon_{3}$ \\
\hline \multirow[t]{2}{*}{$\mathrm{G}(3)$} & $\delta_{1}-\epsilon_{1}, \delta_{1}+\epsilon_{2}, \delta_{1}-\epsilon_{3}$ & \multirow{2}{*}{$\begin{array}{l}\epsilon_{2} \\
\epsilon_{3}-\epsilon_{2}\end{array}$} \\
\hline & $\delta_{1}-\epsilon_{2}, \delta_{1}+\epsilon_{3}$, & \\
\hline
\end{tabular}
simple root $\alpha_{s}$ as follows:

Notice that $\mathrm{B}(0, n)$ has no root of length zero. Thus we complete the proof.

Lemma 2.23. For $\alpha \in \Delta$, we have

$$
\alpha\left(H_{\alpha}\right)=\left\{\begin{array}{ll}
2 & \text { if }(\alpha, \alpha) \neq 0 \\
0 & \text { if }(\alpha, \alpha)=0
\end{array} .\right.
$$


Proof. By (9), we may assume that $\alpha \in \Delta^{+}$. If $\alpha \in \mathrm{W}\left(\Delta_{\overline{0}} \cap \Pi\right)$, then the lemma follows from Proposition 2.21. If $(\alpha, \alpha)=0$, then the lemma is a consequence of Proposition 2.21 and Lemma 2.22. If $\alpha \in \Delta_{\overline{1}}^{+}$such that $2 \alpha \in \Delta_{\overline{0}}^{+}$, (i.e., $(\alpha, \alpha) \neq 0)$, then one can check that $2 \alpha \in \Delta_{\overline{0}}^{+} \backslash \mathrm{W}\left(\Delta_{\overline{0}} \cap \Pi\right)$. Hence it is enough to prove the lemma for $\alpha \in \Delta_{\overline{0}}^{+} \backslash \mathrm{W}\left(\Delta_{\overline{0}} \cap \Pi\right)$.

Now let us fix $\beta$ as in the proof of Proposition 2.21. Then it is easy to see that $\alpha \in \mathrm{W} \beta$, and hence the lemma again follows from Proposition 2.21, since in these cases, one can easily check that $\beta\left(H_{\beta}\right)=2$.

\section{Chevalley basis of basic classical Lie algebras}

In this section, we will show the existence of a Chevalley basis of the basic classical Lie superalgebras $\mathfrak{g}$ in a way similar to [Che]. (In the case of $\mathrm{A}(1,1)$, we once consider the contragradient Lie superalgebra $\tilde{\mathfrak{g}}$ instead of $\mathfrak{g}$ and take the projection of its derived subalgebra.) For later use, we also present a Chevalley bases of $\overline{\mathfrak{g}}$.

For $\alpha=\sum_{i \in \mathrm{I}} k_{i} \alpha_{i} \in \Delta$, we set

$$
\sigma_{\alpha}:=\left\{\begin{array}{cl}
-1 & \text { if } \alpha \in-\Delta_{\overline{1}}^{+} \\
1 & \text { otherwise }
\end{array},\right.
$$

and by definition we have

$$
\sigma_{-\alpha}=(-1)^{|\alpha|} \sigma_{\alpha}
$$

For each $\alpha \in \Delta$, choose a root vector $X_{\alpha} \in \mathfrak{g}_{\alpha}$ so that they satisfy

$$
\left[X_{\alpha}, X_{-\alpha}\right]=\sigma_{\alpha} H_{\alpha}
$$

then we have for these root vectors, we define $N_{\alpha, \beta} \in \mathbb{C}(\alpha, \beta \in \Delta)$ by

$$
N_{\alpha, \beta}:= \begin{cases}\text { the coefficient of } X_{\alpha+\beta} \text { in }\left[X_{\alpha}, X_{\beta}\right] & \text { if } \alpha+\beta \in \Delta \\ 0 & \text { if } \alpha+\beta \notin \Delta\end{cases}
$$

By direct calculation, we have

Lemma 3.1. For each $\alpha \in \Delta$, we take a non-zero numbers $u_{\alpha} \in \mathbb{C}$ and set $X_{\alpha}^{\prime}:=u_{\alpha} X_{\alpha}$. Using $\left\{X_{\alpha}^{\prime}\right\}$, we define the structure constants $\left\{N_{\alpha, \beta}^{\prime}\right\}$ as in (13). Suppose that $\left[X_{\alpha}^{\prime}, X_{-\alpha}^{\prime}\right]=\sigma_{\alpha} H_{\alpha}$. Then we have $u_{\alpha} u_{-\alpha}=1$ and $N_{\alpha, \beta}^{\prime}=$ $u_{\alpha} u_{\beta} u_{\alpha+\beta}^{-1} N_{\alpha, \beta}$.

This lemma says that $N_{\alpha, \beta} N_{-\alpha,-\beta}$ does not depend on the choice of the root vectors that satisfy the condition (12). Thus we first compute $N_{\alpha, \beta} N_{-\alpha,-\beta}$.

We fix $\alpha, \beta \in \Delta$. Here we consider the following three cases:

Case 1: $\alpha \in \Delta_{\overline{0}}$. 
Case 2: $\alpha \in \Delta_{\overline{1}}$ such that $(\alpha, \alpha) \neq 0$.

Case 3: $\alpha \in \Delta_{\overline{1}}$ such that $(\alpha, \alpha)=0$.

Let $p$ and $q$ be positive integers such that

$$
\beta+i \alpha \in \Delta \sqcup\{0\} \quad \text { if and only if } \quad-p \leq i \leq q .
$$

Lemma 3.2. In the above three cases, we have

Case 1: $N_{\alpha, \beta} N_{-\alpha, \alpha+\beta}=q(p+1)$.

Case 2: $N_{\alpha, \beta} N_{-\alpha, \alpha+\beta}=\sigma_{\alpha} \times\left\{\begin{array}{cl}-q & \text { if } p \equiv 0(\bmod 2) \\ p+1 & \text { if } p \equiv 1(\bmod 2)\end{array}\right.$.

Case 3: $N_{\alpha, \beta} N_{-\alpha, \alpha+\beta}=\sigma_{\alpha} \beta\left(H_{\alpha}\right) \delta_{p, 0}^{(2)}$, where

$$
\delta_{p, 0}^{(2)}=\left\{\begin{array}{ll}
1 & \text { if } p \equiv 0(\bmod 2) \\
0 & \text { if } p \equiv 1(\bmod 2)
\end{array} .\right.
$$

Proof. By the Jacobi identity

$$
\left[\left[X_{\alpha}, X_{-\alpha}\right], X_{\beta}\right]=\left[X_{\alpha},\left[X_{-\alpha}, X_{\beta}\right]\right]+(-1)^{|\alpha||\beta|}\left[\left[X_{\alpha}, X_{\beta}\right], X_{-\alpha}\right],
$$

we have

$$
\sigma_{\alpha} \beta\left(H_{\alpha}\right)=N_{\alpha, \beta-\alpha} N_{-\alpha, \beta}+(-1)^{|\alpha||\beta|} N_{\alpha, \beta} N_{\alpha+\beta,-\alpha} .
$$

Using (14), we will show the lemma in each case.

For Case 1, (14) can be written as

$$
\beta\left(H_{\alpha}\right)+N_{\alpha, \beta-\alpha} N_{\beta,-\alpha}=N_{\alpha, \beta} N_{\alpha+\beta,-\alpha} .
$$

Replacing $\beta$ to $\beta-i \alpha$ for $0 \leq i \leq p$, we consider the following summation:

$$
\sum_{i=0}^{p}\left\{(\beta-i \alpha)\left(H_{\alpha}\right)+N_{\alpha, \beta-(i+1) \alpha} N_{\beta-i \alpha,-\alpha}\right\}=\sum_{i=0}^{p} N_{\alpha, \beta-i \alpha} N_{\beta-(i-1) \alpha,-\alpha} .
$$

By direct computation, we obtain

$$
(p+1) \beta\left(H_{\alpha}\right)-\frac{1}{2} p(p+1) \alpha\left(H_{\alpha}\right)+N_{\alpha, \beta-(p+1) \alpha} N_{\beta-p \alpha,-\alpha}=N_{\alpha, \beta} N_{\alpha+\beta,-\alpha} .
$$

Notice that $\beta\left(H_{\alpha}\right)=p-q$, and $\beta-(p+1) \alpha \notin \Delta$ i.e. $N_{\alpha, \beta-(p+1) \alpha}:=0$. Hence the lemma follows from Lemma 2.23 for Case 1 .

For Case 2, (14) can be written as

$$
\sigma_{\alpha} \beta\left(H_{\alpha}\right)+(-1)^{|\beta|} N_{\alpha, \beta-\alpha} N_{\beta,-\alpha}=(-1)^{|\beta|} N_{\alpha, \beta} N_{\alpha+\beta,-\alpha} .
$$

Similarly to Case 1 , we consider the following summation:

$$
\begin{gathered}
\sum_{i=0}^{p}(-1)^{i}\left\{\sigma_{\alpha}(\beta-i \alpha)\left(H_{\alpha}\right)+(-1)^{|\beta-i \alpha|} N_{\alpha, \beta-(i+1) \alpha} N_{\beta-i \alpha,-\alpha}\right\} \\
=\sum_{i=0}^{p}(-1)^{|\beta-i \alpha|+i} N_{\alpha, \beta-i \alpha} N_{\beta-(i-1) \alpha,-\alpha} .
\end{gathered}
$$


Then we have

$\sigma_{\alpha} \sum_{i=0}^{p}(-1)^{i}(\beta-i \alpha)\left(H_{\alpha}\right)+(-1)^{|\beta|} N_{\alpha, \beta-(p+1) \alpha} N_{\beta-p \alpha,-\alpha}=(-1)^{|\beta|} N_{\alpha, \beta} N_{\alpha+\beta,-\alpha}$.

By Lemma 2.23, we obtain

$$
\sum_{i=0}^{p}(-1)^{i}(\beta-i \alpha)\left(H_{\alpha}\right)=\left\{\begin{array}{cl}
-q & \text { if } p \equiv 0(\bmod 2) \\
p+1 & \text { if } p \equiv 1(\bmod 2)
\end{array}\right.
$$

and hence we have the desired result.

For Case 3, by the same argument as in Case 2, we have

$$
\sigma_{\alpha} \sum_{i=0}^{p}(-1)^{i}(\beta-i \alpha)\left(H_{\alpha}\right)=(-1)^{|\beta|} N_{\alpha, \beta} N_{\alpha+\beta,-\alpha} .
$$

Thus Lemma 2.23 implies the conclusion for Case 3 and completes the proof.

\section{Lemma 3.3.}

$$
N_{-\alpha, \alpha+\beta}=(-1)^{|\alpha|} \frac{\sigma_{\alpha+\beta}}{\sigma_{\beta}} \frac{\varepsilon_{\alpha+\beta}}{\varepsilon_{\beta}} N_{-\beta,-\alpha}
$$

Proof. The Jacobi identity

$$
\left[\left[X_{-\alpha}, X_{-\beta}\right], X_{\alpha+\beta}\right]=\left[X_{-\alpha},\left[X_{-\beta}, X_{\alpha+\beta}\right]\right]+(-1)^{|\beta|(|\alpha|+|\beta|)}\left[\left[X_{-\alpha}, X_{\alpha+\beta}\right], X_{-\beta}\right]
$$

implies that

$$
N_{-\alpha,-\beta} \sigma_{-\alpha-\beta} H_{-\alpha-\beta}=N_{-\beta, \alpha+\beta} \sigma_{-\alpha} H_{-\alpha}+(-1)^{|\beta|(|\alpha|+|\beta|)} N_{-\alpha, \alpha+\beta} \sigma_{\beta} H_{\beta} .
$$

Combining (11) and (9), we have

$$
\begin{aligned}
(-1)^{|\beta||\alpha|+|\beta|+|\alpha|} N_{-\beta,-\alpha} \sigma_{\alpha+\beta} H_{\alpha+\beta}= & -(-1)^{|\alpha|} N_{-\beta, \alpha+\beta} \sigma_{\alpha} H_{\alpha} \\
& +(-1)^{|\beta|(|\alpha|+|\beta|)} N_{-\alpha, \alpha+\beta} \sigma_{\beta} H_{\beta} .
\end{aligned}
$$

On the other hand, by definition, we have

$$
\varepsilon_{\alpha+\beta}^{-1} H_{\alpha+\beta}=\varepsilon_{\alpha}^{-1} H_{\alpha}+\varepsilon_{\beta}^{-1} H_{\beta}
$$

for $\alpha, \beta, \alpha+\beta \in \Delta$.

In the case where $H_{\alpha}$ and $H_{\beta}$ are linearly independent, substituting $H_{\alpha+\beta}$ in (15) by (16) and comparing the coefficients of $H_{\beta}$, we obtain the conclusion.

In the case where $H_{\alpha}$ and $H_{\beta}$ are linearly dependent, if $\alpha, \beta, \alpha+\beta \in \Delta$, then $\beta=-\frac{1}{2} \alpha, \alpha$ or $-2 \alpha$. From the facts that $\frac{1}{4} \varepsilon_{\frac{1}{2} \alpha}=\varepsilon_{\alpha}=4 \varepsilon_{2 \alpha}$ and $\frac{1}{2} H_{\frac{1}{2} \alpha}=H_{\alpha}=2 H_{2 \alpha}$, the lemma is proved.

Lemma 3.4. Suppose that $\alpha, \beta \in \Delta$ such that $\alpha+\beta \in \Delta$. Then we have 
L1.Case: $\alpha \in \Delta_{\overline{0}}$ or $\beta \in \Delta_{\overline{0}}$,

$$
N_{\alpha, \beta} N_{-\beta,-\alpha}=\frac{\sigma_{\alpha} \sigma_{\beta}}{\sigma_{\alpha+\beta}}(p+1)^{2}
$$

L2.Case: $\alpha, \beta \in \Delta_{\overline{1}}$ such that $(\alpha, \alpha) \neq 0$ or $(\beta, \beta) \neq 0$,

$$
N_{\alpha, \beta} N_{-\beta,-\alpha}=-\frac{\sigma_{\alpha} \sigma_{\beta}}{\sigma_{\alpha+\beta}}(p+1)^{2} .
$$

L3.Case: $\alpha, \beta \in \Delta_{\overline{1}}$ such that $(\alpha, \alpha)=0$ and $(\beta, \beta)=0$, we have

$$
N_{\alpha, \beta} N_{-\beta,-\alpha}=-\frac{\sigma_{\alpha} \sigma_{\beta}}{\sigma_{\alpha+\beta}} \beta\left(H_{\alpha}\right)^{2} .
$$

Proof. If $\alpha$ and $\beta$ are linearly dependent, then the lemma follows from the proof of Lemma 3.3. Hence we may assume that $\alpha$ and $\beta$ are linearly independent. Since certain combinations of the roots $\alpha$ and $\beta$ form the root system of a rank two basic classical Lie superalgebra, we can reduce the proof of these formulas to the rank two cases. The following lemma completes the proof.

Lemma 3.5. Let $\mathfrak{g}$ be a rank two basic classical Lie superalgebra (rank two Lie algebras $\mathrm{A}_{2}, \mathrm{~B}_{2}$ and $\mathrm{G}_{2}$ are included) and $\Delta$ the root system of $\mathfrak{g}$. Then for $\alpha, \beta \in \Delta$ such that $\alpha+\beta \in \Delta$, the formulas (17), (18) or (19) hold.

Proof. Since in the case of $\mathrm{A}_{2}, \mathrm{~B}_{2}$ and $\mathrm{G}_{2}$, the lemma is proved in [Che], we may assume that $\mathfrak{g}$ is not of type $\mathrm{A}_{2}, \mathrm{~B}_{2}$ and $\mathrm{G}_{2}$.

First we notice that in the case of $\mathbf{L 1}$ (resp. L2), we may assume that $\alpha \in \Delta_{\overline{0}}$ (resp. $\alpha \in \Delta_{\overline{1}}$ ) such that $(\alpha, \alpha) \neq 0$ without loss of generality. Under this assumption, we will show that

$$
\begin{aligned}
& \text { L1. } N_{-\alpha, \alpha+\beta}=\frac{\sigma_{\alpha+\beta}}{\sigma_{\beta}} q(p+1)^{-1} N_{-\beta,-\alpha} . \\
& \text { L2. } N_{-\alpha, \alpha+\beta}=(-1)^{p} \frac{\sigma_{\alpha+\beta}}{\sigma_{\beta}} q(p+1)^{-1} N_{-\beta,-\alpha} . \\
& \text { L3. } N_{-\alpha, \alpha+\beta}=-\frac{\sigma_{\alpha+\beta}}{\sigma_{\beta}} \beta\left(H_{\alpha}\right)^{-1} N_{-\beta,-\alpha} .
\end{aligned}
$$

As in [Che], we can reduce to the following cases:

L1. $\alpha \in \Pi \cap \Delta_{\overline{0}}$ and $\beta \in \Delta^{+}$such that $\alpha+\beta \in \Delta$.

L2. $\alpha \in \Pi \cap \Delta_{\overline{1}}$ such that $(\alpha, \alpha) \neq 0$ and $\beta \in \Delta_{\overline{1}}^{+}$such that $\alpha+\beta \in \Delta$.

L3. $\alpha \in \Pi \cap \Delta_{\overline{1}}$ and $\beta \in \Delta_{\overline{1}}^{+}$such that $(\alpha, \alpha)=0=(\beta, \beta)$ and $\alpha+\beta \in \Delta$.

The following table shows the complete list of such $\alpha$ and $\beta$ : 


\begin{tabular}{|c|c|c|}
\hline Dynkin & $\left\{\varepsilon_{\alpha} \mid \alpha \in \Delta^{+}\right\}$ & $(\alpha, \beta)$ \\
\hline$\bigcirc_{\lambda}^{\alpha_{1}}{ }^{\alpha_{2}}$ & $\varepsilon_{\alpha_{1}}= \pm 1, \varepsilon_{\alpha_{2}}=\varepsilon_{\alpha_{1}+\alpha_{2}}=\mp \lambda$ & $\left(\alpha_{1}, \alpha_{2}\right)$ \\
\hline$\stackrel{\alpha_{1}}{\alpha_{\lambda}} \stackrel{\alpha_{2}}{\otimes}$ & $\varepsilon_{\alpha_{1}}=\varepsilon_{\alpha_{2}}= \pm \lambda, \varepsilon_{\alpha_{1}+\alpha_{2}}= \pm 1$ & $\left(\alpha_{1}, \alpha_{2}\right)$ \\
\hline $\begin{array}{l}\alpha_{1} \quad \alpha_{2} \\
\bigcirc=\end{array}$ & $\begin{array}{l}\varepsilon_{\alpha_{1}}=2, \varepsilon_{\alpha_{2}}=\varepsilon_{2 \alpha_{1}+\alpha_{2}}=-1, \\
\varepsilon_{\alpha_{1}+\alpha_{2}}=-2, \varepsilon_{2 \alpha_{1}+2 \alpha_{2}}=-\frac{1}{2}\end{array}$ & $\begin{array}{l}\left(\alpha_{1}, \alpha_{2}\right),\left(\alpha_{1}, \alpha_{1}+\alpha_{2}\right) \\
\left(\alpha_{2}, 2 \alpha_{1}+\alpha_{2}\right)\end{array}$ \\
\hline $\begin{array}{l}\alpha_{1} \quad \alpha_{2} \\
\Longleftarrow=1\end{array}$ & $\begin{array}{l}\varepsilon_{\alpha_{1}}=-2, \varepsilon_{\alpha_{2}}=\varepsilon_{2 \alpha_{1}+\alpha_{2}}=-1 \\
\varepsilon_{\alpha_{1}+\alpha_{2}}=2, \varepsilon_{2 \alpha_{1}}=-\frac{1}{2}\end{array}$ & $\left(\alpha_{1}, \alpha_{2}\right)$ \\
\hline$\stackrel{\alpha_{1}}{\alpha_{2}}$ & $\begin{array}{l}\varepsilon_{\alpha_{1}}=\varepsilon_{\alpha_{1}+2 \alpha_{2}}=1, \varepsilon_{\alpha_{2}}=\varepsilon_{\alpha_{1}+\alpha_{2}}=2 \\
\varepsilon_{2 \alpha_{2}}=\varepsilon_{2 \alpha_{1}+2 \alpha_{2}}=\frac{1}{2}\end{array}$ & $\begin{array}{l}\left(\alpha_{1}, \alpha_{2}\right),\left(\alpha_{1}, \alpha_{1}+2 \alpha_{2}\right), \\
\left(\alpha_{1}, 2 \alpha_{2}\right),\left(\alpha_{2}, \alpha_{1}+\alpha_{2}\right) \\
\end{array}$ \\
\hline
\end{tabular}

Now the above formula can be shown by case by case checking and the lemma follows from Lemma 3.2 .

Remark 3.3.6. For each basic classical Lie superalgebra $\mathfrak{g}, \lambda$ in the above proof is given as follows:

\begin{tabular}{|c|c||c|c|}
\hline type of $\mathfrak{g}$ & $\lambda$ & type of $\mathfrak{g}$ & $\lambda$ \\
\hline $\mathrm{A}(m, n)$ & \pm 1 & $\mathrm{D}(2,1 ; a)$ & $-1, a+1,-a$ \\
\hline $\mathrm{C}(n)$ & $-1,-2$ & $\mathrm{~F}(4)$ & $-1,-2,3$ \\
\hline $\mathrm{B}(m, n)$ & $\pm 1,2$ & $\mathrm{G}(3)$ & $-\frac{1}{2},-\frac{3}{2}, 2$ \\
\hline $\mathrm{D}(m, n)$ & $\pm 1,-2$ & & \\
\hline
\end{tabular}

Let $\theta: \mathfrak{g} \rightarrow \mathfrak{g}$ be an anti-involution of $\mathfrak{g}$ such that $\theta\left(\mathfrak{g}_{\alpha}\right) \subset \mathfrak{g}_{-\alpha}$ and let $c_{\alpha}$ $(\alpha \in \Delta)$ be a non-zero constant such that $\theta X_{\alpha}=c_{\alpha} X_{-\alpha}$

Lemma 3.7. We have $\left.\theta\right|_{\mathfrak{h}}=\mathrm{i} d_{\mathfrak{h}}$ and $c_{\alpha} c_{-\alpha}=1$ for any $\alpha \in \Delta$.

Proof. We first show the first assertion. For $H \in \mathfrak{h}$, by applying $\theta$ to $\left[H, X_{\beta}\right]=$ $\beta(H) X_{\beta}$, we obtain

$$
\left[\theta X_{\beta}, \theta H\right]=\beta(H) \theta X_{\beta} .
$$

Since $\theta X_{\beta} \in \mathfrak{g}_{-\beta}$, we conclude that $\theta H=H$.

Next we show the second assertion. By applying $\theta$ to $\left[X_{\alpha}, X_{-\alpha}\right]=\sigma_{\alpha} H_{\alpha}$, we have

$$
\left[\theta X_{-\alpha}, \theta X_{\alpha}\right]=c_{\alpha} c_{-\alpha}\left[X_{\alpha}, X_{-\alpha}\right]=\sigma_{\alpha} H_{\alpha}
$$


Thus we obtain $c_{\alpha} c_{-\alpha}=1$.

In the sequel, for each $\alpha \in \Delta$ let us take a non-zero constant $u_{ \pm \alpha} \in \mathbb{C}$ as

$$
u_{\alpha}^{2}=\left\{\begin{array}{cl}
c_{\alpha} & \text { if } \alpha \in \Delta_{\overline{0}} \\
-\sqrt{-1} \sigma_{\alpha} c_{\alpha} & \text { if } \alpha \in \Delta_{\overline{1}}
\end{array}, \quad \text { and } u_{\alpha} u_{-\alpha}=1 .\right.
$$

Lemma 3.8. If we set $\tilde{X}_{\alpha}:=u_{\alpha}^{-1} X_{\alpha}$ for each $\alpha \in \Delta$, then we have

$$
\theta \tilde{X}_{\alpha}=\left\{\begin{array}{ll}
\tilde{X}_{-\alpha} & \text { if }|\alpha|=0 \\
\sqrt{-1} \sigma_{\alpha} \tilde{X}_{-\alpha} & \text { if }|\alpha|=1
\end{array} .\right.
$$

Proof. For $\alpha \in \Delta_{\overline{0}}$, we have

$$
\theta \tilde{X}_{\alpha}=u_{\alpha}^{-1} c_{\alpha} X_{-\alpha}=u_{\alpha} X_{-\alpha}=u_{-\alpha}^{-1} X_{-\alpha}=\tilde{X}_{-\alpha} .
$$

For $\alpha \in \Delta_{\overline{1}}$, we have

$$
\theta \tilde{X}_{\alpha}=u_{\alpha}^{-1} c_{\alpha} X_{-\alpha}=\sqrt{-1} \sigma_{\alpha} u_{\alpha} X_{-\alpha}=\sqrt{-1} \sigma_{\alpha} u_{-\alpha}^{-1} X_{-\alpha}=\sqrt{-1} \sigma_{\alpha} \tilde{X}_{-\alpha} .
$$

Hence we have proved the lemma.

From Lemma 3.8, we may assume that the root vectors $\left\{X_{\alpha}\right\}$ satisfy the formulas (20). Applying $\theta$ to the both sides of $\left[X_{\alpha}, X_{\beta}\right]=N_{\alpha, \beta} X_{\alpha+\beta}$, we have

$$
\sigma_{\alpha} \sigma_{\beta}(-1)^{\frac{1}{2}(|\alpha|+|\beta|)} N_{-\beta,-\alpha} X_{-\alpha-\beta}=(-1)^{\frac{1}{2}|\alpha+\beta|} N_{\alpha, \beta} \sigma_{\alpha+\beta} X_{-\alpha-\beta} .
$$

Hence we have

$$
N_{-\beta,-\alpha}=(-1)^{\frac{1}{2}(|\alpha+\beta|-|\alpha|-|\beta|)} \frac{\sigma_{\alpha+\beta}}{\sigma_{\alpha} \sigma_{\beta}} N_{\alpha, \beta}
$$

Combining this formula with Lemma 3.4, we obtain the main result of this section, that can be stated as follows:

\section{Theorem 3.9.}

1. Let $\mathfrak{g}$ be a basic classical Lie superalgebra over $\mathbb{C}$. For each $\alpha \in \Delta$, we define $\sigma_{\alpha}$ and $H_{\alpha}$ as in (10) and (8). Then there exist root vectors $\left\{X_{\alpha} \in \mathfrak{g}_{\alpha} \mid \alpha \in \Delta\right\}$ such that

I) $\left[X_{\alpha}, X_{-\alpha}\right]=\sigma_{\alpha} H_{\alpha}$,

II) $\left[X_{\alpha}, X_{\beta}\right]=N_{\alpha, \beta} X_{\alpha+\beta}$,

where the structure constants $\left\{N_{\alpha, \beta}\right\}$ satisfy the following conditions:

i) If $\alpha \in \Delta_{\overline{0}}$ or $\beta \in \Delta_{\overline{0}}$ (we assume that $\alpha \in \Delta_{\overline{0}}$ ) and $\alpha+\beta \in \Delta$, then

$$
N_{\alpha, \beta}^{2}=(p+1)^{2} \text {. }
$$

ii) If $\alpha, \beta \in \Delta_{\overline{1}}$ satisfy $(\alpha, \alpha) \neq 0$ or $(\beta, \beta) \neq 0$ (we assume that $(\alpha, \alpha) \neq$ 0 ) and $\alpha+\beta \in \Delta$, then

$$
N_{\alpha, \beta}^{2}=(p+1)^{2}
$$


iii) If $\alpha, \beta \in \Delta_{\overline{1}}$ satisfy $(\alpha, \alpha)=0$ and $(\beta, \beta)=0$ and $\alpha+\beta \in \Delta$, then

$$
N_{\alpha, \beta}^{2}=\beta\left(H_{\alpha}\right)^{2} \text {. }
$$

Here $p:=\max \{i \mid \beta-i \alpha \in \Delta\}$.

2. Let $\left\{X_{\alpha}^{\prime} \mid \alpha \in \Delta\right\}$ be another set of root vectors satisfying the above conditions and $\left\{N_{\alpha, \beta}^{\prime} \mid \alpha, \beta \in \Delta\right\}$ be their structure constant defined as (13). Then there exist $\left\{u_{\alpha} \mid \alpha \in \Delta\right\} \subset\{ \pm 1\}$ such that $u_{\alpha} u_{-\alpha}=1$ and

$$
N_{\alpha, \beta}^{\prime}=u_{\alpha} u_{\beta} u_{\alpha+\beta}^{-1} N_{\alpha, \beta},
$$

for any $\alpha, \beta \in \Delta$.

Proof. The second assertion can be proved by the same argument as in [Che].

In the sequel, we call the set $\left\{H_{\alpha}, X_{\alpha} \mid \alpha \in \Delta\right\}$ a Chevalley basis of $\mathfrak{g}$ if it satisfies the conditions in Theorem 3.9.

From the Appendix, we obtain

Corollary 3.10. Let $\mathfrak{g}$ be a basic classical Lie superalgebra over $\mathbb{C}$. In the case where $\mathfrak{g}$ is of type $\mathrm{D}(2,1 ; a)$, we assume that $a \in \mathbb{Z} \backslash\{0,-1\}$. Then there exists a $\mathbb{Z}$-form $\mathfrak{g}_{\mathbb{Z}}$ of $\mathfrak{g}$.

Thus for a basic classical Lie superalgebra $\mathfrak{g}$ satisfying the conditions in Corollary 3.10 , we can define the Lie superalgebra $\mathfrak{g}_{k}$ over an arbitrary commutative ring $k$ by

$$
\mathfrak{g}_{k}:=k \otimes_{\mathbb{Z}} \mathfrak{g}_{\mathbb{Z}}
$$

Here we remark on rank two subalgebras of basic classical Lie superalgebras.

Remark 3.3.11. There exist the following isomorphisms of corresponding Lie superalgebras $\mathfrak{g}_{\mathbb{Z}}$ over $\mathbb{Z}$ :

$$
\bigcirc_{\lambda} \otimes \simeq \bigcirc_{-\lambda} \otimes \simeq \bigotimes \frac{-\lambda}{-\lambda} \otimes \frac{-\lambda}{-\lambda} \otimes
$$

Finally, we define a Chevalley basis of the Lie superalgebra $\overline{\mathfrak{g}}$, for type $\mathrm{A}(n, n)$. In the case where $\overline{\mathfrak{g}}=\mathfrak{s l}(n+1, n+1)$ for $n \geq 2$, from the proof of Theorem 3.9 , there exists a Chevalley basis, i.e., coroots $\left\{H_{\alpha} \mid \alpha \in \Delta\right\}$ and root vectors $\left\{X_{\alpha} \mid \alpha \in \Delta\right\}$ which satisfy the conditions in Theorem 3.9. As stated before, we use the same notation to denote a Chevalley basis of $\mathfrak{g}$ and $\overline{\mathfrak{g}}$. In the case where $\overline{\mathfrak{g}}=\mathfrak{d}$, for the basis of $\mathfrak{d}$ defined as in (2), the structure constants are integers. Though it does not satisfy the conditions in Theorem 3.9, we also call this basis the Chevalley basis of $\mathfrak{d}$. We have 
Lemma 3.12. Let $\mathfrak{g}$ be a basic classical Lie superalgebra of type $\mathrm{A}(n, n)$ for some $n$. Then there exists a $\mathbb{Z}$-form $\overline{\mathfrak{g}}_{\mathbb{Z}}$ of the Lie superalgebra $\overline{\mathfrak{g}}$.

For a commutative ring $k$, we also define the Lie superalgebra $\overline{\mathfrak{g}}_{k}$ over $k$ by

$$
\overline{\mathfrak{g}}_{k}:=k \otimes_{\mathbb{Z}} \overline{\mathfrak{g}}_{\mathbb{Z}} .
$$

\section{Universal central extensions}

\subsection{General remarks}

In the first subsection, we collect definitions and general properties of central extensions of Lie superalgebras.

Let $k$ be a commutative ring and $\mathfrak{a}$ a Lie superalgebra over $k$. From now on, we assume that $\frac{1}{2} \in k$.

To define the universal central extension of $\mathfrak{a}$, we recall some definitions. We say that a Lie superalgebra $\mathfrak{a}$ is perfect if $\mathfrak{a}=[\mathfrak{a}, \mathfrak{a}]$. A short exact sequence of Lie superalgebras

$$
0 \longrightarrow \mathfrak{c} \longrightarrow \mathfrak{u} \stackrel{\alpha}{\longrightarrow} \mathfrak{a} \longrightarrow 0
$$

is called a central extension of $\mathfrak{a}$ by $\mathfrak{c}$ if $\mathfrak{c}$ is a commutative Lie algebra over $k$ i.e. $\mathfrak{c}_{\overline{1}}=\{0\}$ and $[\mathfrak{c}, \mathfrak{u}]=0 . \mathfrak{c}$ is called the kernel of the central extension (22). We sometimes denote the above central extension by $\alpha: \mathfrak{u} \rightarrow \mathfrak{a}$.

Next we introduce an equivalence relation between central extensions. Let $\mathrm{V}$ be a $k$-module. Two central extensions

$$
\begin{aligned}
& 0 \longrightarrow \mathrm{V} \longrightarrow \mathfrak{a}_{1} \longrightarrow \mathfrak{a} \longrightarrow 0, \\
& 0 \longrightarrow \mathrm{V} \longrightarrow \mathfrak{a}_{2} \longrightarrow \mathfrak{a} \longrightarrow 0
\end{aligned}
$$

are said to be equivalent if there exists a homomorphism of Lie superalgebras from $\mathfrak{a}_{1}$ to $\mathfrak{a}_{2}$ such that the diagram

$$
\begin{aligned}
& \| \mathrm{V} \\
& 0 \rightarrow \mathrm{V} \rightarrow \mathfrak{a}_{1} \rightarrow \mathfrak{a} \rightarrow 0 \\
& \downarrow
\end{aligned} \underset{\mathfrak{a}}{\downarrow} \rightarrow 0
$$

commutes. The set of equivalence classes of such central extensions are known to be parameterized by the second cohomology group $H^{2}(\mathfrak{a}, \mathrm{V})$. To be precise, we first introduce $Z^{2}(\mathfrak{a}, V)$ and $B^{2}(\mathfrak{a}, V)$ as follows. Set

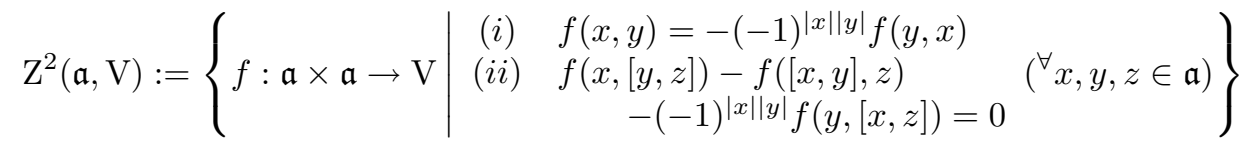

and

$$
\mathrm{B}^{2}(\mathfrak{a}, \mathrm{V}):=\left\{\begin{array}{l|l}
f: \mathfrak{a} \times \mathfrak{a} \rightarrow \mathrm{V} \mid \begin{array}{l}
f(x, y)=g([x, y]) \\
\text { for some } k \text {-linear map } g: \mathfrak{a} \rightarrow \mathrm{V}
\end{array}
\end{array}\right\}
$$


Lemma 4.1. Let $\mathrm{V}$ be a $k$-module. The second cohomology group $H^{2}(\mathfrak{a}, \mathrm{V}):=$ $\mathrm{Z}^{2}(\mathfrak{a}, \mathrm{V}) / \mathrm{B}^{2}(\mathfrak{a}, \mathrm{V})$ are in one-to-one correspondence with the set of the equivalence classes of the central extensions of $\mathfrak{a}$ by $\mathrm{V}$.

Proof. First we notice that for each central extension $0 \rightarrow \mathrm{V} \rightarrow \mathfrak{a}_{1} \rightarrow \mathfrak{a} \rightarrow 0$, one may associate a two cocycle $\mathrm{F} \in \mathrm{Z}^{2}(\mathfrak{a}, \mathrm{V})$. Indeed, for $x, y \in \mathfrak{a}$, if we set

$$
\mathrm{F}(x, y):=[(x, 0),(y, 0)]-([x, y], 0) \in \mathfrak{a}_{1},
$$

then we have $\mathrm{F}(x, y) \in \mathrm{V}$ and $\mathrm{F}$ satisfies the 2 -cocycle conditions.

Conversely, for each $f \in \mathrm{Z}^{2}(\mathfrak{a}, \mathrm{V})$, one can define a central extension

$$
0 \longrightarrow \mathrm{V} \longrightarrow \mathfrak{a}_{f} \longrightarrow \mathfrak{a} \longrightarrow 0,
$$

by

$$
[(x, v),(y, w)]_{f}:=([x, y], f(x, y))
$$

where $x, y \in \mathfrak{a}$ and $u, w \in \mathrm{V}$.

Let $f$ and $g$ be elements of $\mathrm{Z}^{2}(\mathfrak{a}, \mathrm{V})$ such that $f-g \in \mathrm{B}^{2}(\mathfrak{a}, \mathrm{V})$ i.e. $(f-$ $g)(x, y)=h([x, y])$, where $h: \mathfrak{a} \rightarrow \mathrm{V}$ is some $k$-linear map. Now we prove that the extensions defined by $f$ and $g$ are equivalent. Let us define $\Phi: \mathfrak{a}_{f} \rightarrow \mathfrak{a}_{g}$ by

$$
\Phi((x, v)):=(x, v-h(x)) .
$$

It is clear that $\Phi$ is bijective. Let us check that $\Phi$ is a homomorphism of Lie superalgebras. We have

$$
\begin{aligned}
{[\Phi((x, v)), \Phi((y, w))]_{g} } & =[(x, v-h(x)),(y, w-h(y))]_{g} \\
& =([x, y], g(x, y)) \\
& =([x, y], f(x, y)-h([x, y])) \\
& =\Phi(([x, y], f(x, y))) \\
& =\Phi\left([(x, v),(y, w)]_{f}\right) .
\end{aligned}
$$

Next we show that for $f, g \in \mathrm{Z}^{2}(\mathfrak{a}, \mathrm{V})$ such that the central extensions $\mathfrak{a}_{f} \rightarrow \mathfrak{a}$ and $\mathfrak{a}_{g} \rightarrow \mathfrak{a}$ are equivalent, we have $f-g \in \mathrm{B}^{2}(\mathfrak{a}, \mathrm{V})$. Let $\Phi$ be a homomorphism of Lie superalgebras such that

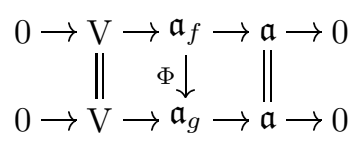

commutes. We can express $\Phi(x, v)=(x, v-h(x))$ for some $k$-linear map $h$ : $\mathfrak{a} \rightarrow \mathrm{V}$. Then we have

$$
\begin{aligned}
\Phi\left([(x, v),(y, w)]_{f}\right) & =\Phi(([x, y], f(x, y))) \\
& =([x, y], f(x, y)-h([x, y])), \\
{[\Phi((x, v)), \Phi((y, w))]_{g} } & =[(x, v-h(x)),(y, w-h(y))]_{g} \\
& =([x, y], g(x, y))
\end{aligned}
$$


and thus $(f-g)(x, y)=h([x, y])$, i.e., $f-g \in \mathrm{B}^{2}(\mathfrak{a}, \mathrm{V})$. We have completed the proof.

Now we define the universal central extension of a Lie superalgebra $\mathfrak{a}$.

Definition 4.2. The central extension (22) of $\mathfrak{a}$ is called the universal central extension if the following conditions hold.

1. $\mathfrak{u}$ is perfect.

2. For any central extension $\beta: \mathfrak{b} \rightarrow \mathfrak{a}$, there exists $\gamma: \mathfrak{u} \rightarrow \mathfrak{b}$ such that the following diagram commutes:

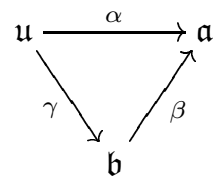

Remark 4.4.3. From the second condition, a universal central extension is unique up to isomorphism of Lie superalgebras.

The following proposition for Lie algebras is proved e.g. in [Gar], [MP].

Proposition 4.4. A Lie superalgebra $\mathfrak{a}$ admits the universal central extension if and only if $\mathfrak{a}$ is perfect.

Proof. Suppose that $\alpha: \mathfrak{u} \rightarrow \mathfrak{a}$ is the universal central extension. By definition, $\mathfrak{u}$ is perfect, and hence

$$
\mathfrak{a}=\alpha(\mathfrak{u})=\alpha([\mathfrak{u}, \mathfrak{u}])=[\alpha(\mathfrak{u}), \alpha(\mathfrak{u})]=[\mathfrak{a}, \mathfrak{a}] .
$$

Next let us suppose that $\mathfrak{a}$ is perfect. We set

$$
\mathrm{W}^{\prime}:=\bigwedge^{2} \mathfrak{a}:=(\mathfrak{a} \otimes \mathfrak{a}) /\left\langle x \otimes y+(-1)^{|x||y|} y \otimes x \mid x, y \in \mathfrak{a}\right\rangle_{k} .
$$

We remark that $\mathrm{W}^{\prime} \simeq \bigwedge^{2} \mathfrak{a}_{\overline{0}} \oplus\left(\mathfrak{a}_{\overline{0}} \bigwedge \mathfrak{a}_{\overline{1}}\right) \oplus \mathrm{S}^{2} \mathfrak{a}_{\overline{0}}$ as a $k$-module. Furthermore, we set

$$
\begin{aligned}
\mathrm{I} & :=\mathrm{B}_{2}(\mathfrak{a}, k) \\
& :=\left\langle x \wedge[y, z]-[x, y] \wedge z-(-1)^{|x||y|} y \wedge[x, z] \mid x, y, z \in \mathfrak{a}\right\rangle_{k}
\end{aligned}
$$

and $\mathrm{W}:=\mathrm{W}^{\prime} / \mathrm{I}$. Let $\omega: \mathrm{W}^{\prime} \rightarrow \mathrm{W}$ be the canonical projection. It is clear that $\omega \in \mathrm{Z}^{2}(\mathfrak{a}, \mathrm{W})$. We consider the central extension

$$
0 \longrightarrow \mathrm{W} \longrightarrow \mathfrak{a}_{\omega} \longrightarrow \mathfrak{a} \longrightarrow 0,
$$

defined by $\omega$. Using this central extension, we construct the universal central extension of $\mathfrak{a}$. Let $\mathrm{V}$ be an arbitrary $k$-module and $f \in \mathrm{Z}^{2}(\mathfrak{a}, \mathrm{V})$. Since 
$f(x, y)=-(-1)^{|x||y|} f(y, x)$, we have a $k$-linear map

$$
\psi^{\prime}: \mathrm{W} \longrightarrow \mathrm{V} \text { such that } \omega(x, y) \mapsto f(x, y) .
$$

Let us define $\phi^{\prime}: \mathfrak{a}_{\omega} \rightarrow \mathfrak{a}_{f}$ by

$$
\phi^{\prime}((x, u)):=\left(x, \psi^{\prime}(u)\right) .
$$

Then it is clear that the diagram

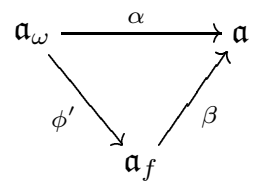

commutes. Now, let us set

$$
\hat{\mathfrak{a}}:=\left[\mathfrak{a}_{\omega}, \mathfrak{a}_{\omega}\right] .
$$

Since $\mathfrak{a}$ is perfect, it follows that $\hat{\mathfrak{a}}+\mathrm{W}=\mathfrak{a}_{\omega}$. This implies that $\hat{\mathfrak{a}}$ is perfect since

$$
\hat{\mathfrak{a}}=[\hat{\mathfrak{a}}+\mathrm{W}, \hat{\mathfrak{a}}+\mathrm{W}]=[\hat{\mathfrak{a}}, \hat{\mathfrak{a}}] .
$$

Furthermore, if we set

$$
\mathfrak{c}:=\mathrm{W} \cap \hat{\mathfrak{a}},
$$

then we have a central extension

$$
0 \longrightarrow \mathfrak{c} \longrightarrow \hat{\mathfrak{a}} \longrightarrow \mathfrak{a} \longrightarrow 0
$$

such that $\hat{\mathfrak{a}}$ is perfect. Now, if we define $\phi$ as the restriction of $\phi^{\prime}$ to the subalgebra $\hat{\mathfrak{a}}$, then the following diagram commutes:

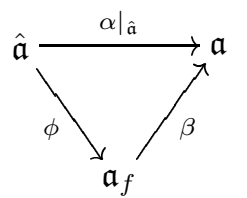

Therefore, $\hat{\mathfrak{a}} \rightarrow \mathfrak{a}$ is the universal central extension and the proof is completed.

As a corollary, we have the following description of the kernel of the universal central extension. For a Lie superalgebra over $k$, let us define

$$
\mathrm{Z}_{2}(\mathfrak{a}, k):=\left\{\sum_{i} x_{i} \wedge y_{i} \in \Lambda^{2} \mathfrak{a} \mid \sum_{i}\left[x_{i}, y_{i}\right]=0\right\} .
$$

In the sequel, we assume that $\mathfrak{a}$ is $k$-free. Under this assumption, from the proof of Proposition 4.4 we obtain

$$
\mathfrak{c}:=\mathrm{W} \cap \hat{\mathfrak{a}}=\left\{\sum_{i} \alpha\left(x_{i}, y_{i}\right) \mid \sum_{i} x_{i} \wedge y_{i} \in \mathrm{Z}_{2}(\mathfrak{a}, k)\right\} / \mathrm{B}_{2}(\mathfrak{a}, k) .
$$


Thus the following lemma holds.

Corollary 4.5. Suppose that a Lie superalgebra $\mathfrak{a}$ over $k$ is $k$-free and perfect. Let $\mathfrak{c}$ be the kernel of the universal central extension of $\mathfrak{a}$. Then we have

$$
\mathfrak{c} \simeq H_{2}(\mathfrak{a}, k)
$$

where $H_{2}(\mathfrak{a}, k):=\mathrm{Z}_{2}(\mathfrak{a}, k) / \mathrm{B}_{2}(\mathfrak{a}, k)$.

Let $\mathrm{A}$ be a commutative $k$-algebra that is a $k$-free module at the same time. As we will see in the next subsection, if $\mathfrak{a}=\mathfrak{g}_{k} \otimes_{k} \mathrm{~A}(\mathfrak{g}$ is a basic classical Lie superalgebra), then $H_{2}(\mathfrak{a}, k)$ is related with the cyclic homology of A. Here we recall the definition of the cyclic homology.

For $n \in \mathbb{Z}_{\geq 0}$, we set

$$
\mathrm{C}_{n}(\mathrm{~A}):= \begin{cases}k & \text { if } n=0 \\ \mathrm{~A} & \text { if } n=1 \\ \mathrm{~A}^{\otimes n} / \mathrm{I}_{n} & \text { if } n>1\end{cases}
$$

where $\mathrm{I}_{n}$ is the $k$-submodule of $\mathrm{A}^{\otimes n}$ generated by

$$
a_{1} \otimes a_{2} \otimes \cdots \otimes a_{n}+(-1)^{n} a_{2} \otimes \cdots \otimes a_{n} \otimes a_{1}, \quad\left(a_{i} \in \mathrm{A}\right) .
$$

Let us define the complex

$$
\begin{aligned}
\mathrm{C}_{*}(\mathrm{~A}) & : \cdots \longrightarrow \mathrm{C}_{n}(\mathrm{~A}) \stackrel{d}{\longrightarrow} \mathrm{C}_{n-1}(\mathrm{~A}) \longrightarrow \cdots \longrightarrow \mathrm{C}_{0}(\mathrm{~A}) \longrightarrow 0 \\
\quad d & : \mathrm{C}_{n}(\mathrm{~A}) \longrightarrow \mathrm{C}_{n-1}(\mathrm{~A})
\end{aligned}
$$

as follows: For $n=0,1$, we set $d:=0$ and for $n>1$,

$$
d\left(a_{1} \otimes a_{2} \otimes \cdots \otimes a_{n}\right):=\sum_{i=2}^{n}(-1)^{i} a_{1} \otimes \cdots \otimes a_{i-1} a_{i} \otimes \cdots \otimes a_{n}-a_{2} \otimes \cdots \otimes a_{n} a_{1} .
$$

Then it turns out that $d$ is well-defined and $d^{2}=0$. The $n$-th homology group of this complex $\mathrm{C}_{*}(\mathrm{~A})$ is denoted by $H \mathrm{C}_{n}(\mathrm{~A})$ and is called the $n$-th cyclic homology of A.

The following proposition is well-known (cf. [Lod]):

Proposition 4.6. Suppose that A is commutative. The following isomorphism exists:

$$
H \mathrm{C}_{2}(\mathrm{~A}) \simeq \Omega_{\mathrm{A} / k}^{1} / d \mathrm{~A}
$$

where $\Omega_{\mathrm{A} / k}^{1} / d \mathrm{~A}$ is the module of Kähler differentials of $\mathrm{A}$ over $k$ modulo exact forms. 


\subsection{Main result}

In this subsection, we will state our main result i.e. the universal central extension of $\mathfrak{g}_{k} \otimes_{k} \mathrm{~A}$. Let us first notice that the Lie superalgebra $\mathfrak{g}_{k} \otimes_{k} \mathrm{~A}$ over $k$ is perfect and hence has the universal central extension

$$
0 \longrightarrow \mathfrak{c} \longrightarrow \mathfrak{g}_{k}(\mathrm{~A}) \longrightarrow \mathfrak{g}_{k} \otimes_{k} \mathrm{~A} \longrightarrow 0 .
$$

Our main theorem can be described as follows:

Theorem 4.7. Let $k$ be a commutative ring and let $\mathrm{A}$ be a $k$-free commutative $k$-algebra. Let $\mathfrak{g}_{k}$ be a basic classical Lie superalgebra over $k$ as was defined in Section 3. Suppose that

$\frac{1}{2} \in k$ if $\mathfrak{g}$ is of type $\mathrm{A}(m, n), \mathrm{C}(n)$ and $\mathrm{D}(m, n)$,

$\frac{1}{2}, \frac{1}{3} \in k$ if $\mathfrak{g}$ is of type $\mathrm{B}(m, n), \mathrm{F}(4)$ and $\mathrm{G}(3)$,

$\frac{1}{2}, \frac{1}{a}, \frac{1}{a+1} \in k$, if $\mathfrak{g}$ is of type $\mathrm{D}(2,1 ; a)$.

Now let us define the Lie superalgebra $\overline{\mathfrak{g}}_{k}$ as follows:

$$
\overline{\mathfrak{g}}_{k}:=\left\{\begin{array}{cl}
\mathfrak{g}_{k} & \text { if } \mathfrak{g} \text { is not of type } \mathrm{A}(n, n) \quad \forall n \\
\mathfrak{d}_{k} & \text { if } \mathfrak{g} \text { is of type } \mathrm{A}(1,1) \\
\mathfrak{s l}(n+1, n+1)_{k} & \text { if } \mathfrak{g} \text { is of type } \mathrm{A}(n, n) \exists n>1
\end{array} .\right.
$$

Then the following hold:

1. $\mathfrak{g}_{k}(\mathrm{~A}) \simeq \overline{\mathfrak{g}}_{k} \otimes_{k} \mathrm{~A} \oplus \Omega_{\mathrm{A} / k}^{1} / d \mathrm{~A}$.

2. The bracket of $\mathfrak{g}_{k}(\mathrm{~A})$ is given by

$$
\begin{gathered}
{[X \otimes a, Y \otimes b]=[X, Y] \otimes a b+(X, Y) \overline{b d a},} \\
a, b \in \mathrm{A}, \quad X, Y \in \overline{\mathfrak{g}}_{k},
\end{gathered}
$$

where $(\cdot, \cdot)$ is an even supersymmetric invariant bilinear form on $\overline{\mathfrak{g}}_{k}$ which is described in Section 2.4, $d: \mathrm{A} \rightarrow \Omega_{\mathrm{A} / k}^{1}$ is the differential and $-: \Omega_{\mathrm{A} / k}^{1} \rightarrow$ $\Omega_{\mathrm{A} / k}^{1} / d \mathrm{~A}$ is the projection.

Remark 4.4.8. If $\mathfrak{g}$ is of type $\mathrm{B}(m, n)$ or $\mathrm{G}(3)$, then $\mathfrak{g}$ has a root $\alpha \in \Delta_{\overline{1}}$ such that $(\alpha, \alpha) \neq 0$, i.e., $\mathfrak{g}$ contains $\mathfrak{o} \mathfrak{s p}(1,2)$ as a subalgebra. In these cases, we have to assume that 3 is invertible in $k$. (See the proof of Lemma 4.13.)

In particular, in the case where $k=\mathbb{C}$ and $\mathrm{A}=\mathbb{C}\left[t, t^{-1}\right]$, we have

Corollary 4.9. Set $\tilde{\mathfrak{g}}:=\mathfrak{g}_{\mathbb{C}}(\mathrm{A})$.

1. $\tilde{\mathfrak{g}} \simeq \overline{\mathfrak{g}}_{\mathbb{C}} \otimes_{\mathbb{C}} \mathbb{C}\left[t, t^{-1}\right] \oplus \mathbb{C} c$.

2. The bracket of $\mathfrak{g}_{\mathbb{C}}(\mathrm{A})$ is given by

$$
\begin{gathered}
{\left[X \otimes t^{r}, Y \otimes t^{s}\right]=[X, Y] \otimes t^{r+s}+(X, Y) r \delta_{r+s, 0} c} \\
r, s \in \mathbb{Z}, \quad X, Y \in \overline{\mathfrak{g}}_{\mathbb{C}}
\end{gathered}
$$


where $(\cdot, \cdot)$ is an even, supersymmetric invariant bilinear form on $\overline{\mathfrak{g}}_{\mathbb{C}}$.

Moreover, from Theorem 4.7, we have

Proposition 4.10. Under the assumption of Theorem 4.7, we have

$$
H_{2}\left(\mathfrak{g}_{k} \otimes_{k} \mathrm{~A}, k\right) \simeq\left\{\begin{array}{ll}
H \mathrm{C}_{2}(\mathrm{~A}) & \text { if } \mathfrak{g} \text { is not of type } \mathrm{A}(n, n) \quad \forall n \\
H \mathrm{C}_{2}(\mathrm{~A}) \oplus \mathrm{A}^{\oplus 3} & \text { if } \mathfrak{g} \text { is of type } \mathrm{A}(1,1) \\
H \mathrm{C}_{2}(\mathrm{~A}) \oplus \mathrm{A} & \text { if } \mathfrak{g} \text { is of type } \mathrm{A}(n, n) \quad \exists n>1
\end{array} .\right.
$$

\subsection{Proof}

We first show the next proposition.

Proposition 4.11. Suppose that $\mathfrak{g}=\mathfrak{s l}_{2}$ or $\mathfrak{o s p}(1,2)$. Let Z be a free $k$ module and

$$
0 \longrightarrow \mathrm{Z} \longrightarrow \mathfrak{g}_{k}^{\prime}(\mathrm{A}) \stackrel{\pi}{\longrightarrow} \mathfrak{g}_{k} \otimes_{k} \mathrm{~A} \longrightarrow 0
$$

a central extension of $\mathfrak{g}_{k} \otimes_{k} \mathrm{~A}$. Then the bracket of $\mathfrak{g}_{k}^{\prime}(\mathrm{A})$ can be described as

$$
[X \otimes a, Y \otimes b]=[X, Y] \otimes a b+(X, Y)\{a, b\},
$$

where $(\cdot, \cdot)$ is a non-degenerate even supersymmetric invariant bilinear form on $\mathfrak{g}_{k},\{\cdot, \cdot\}: \mathrm{A} \times \mathrm{A} \rightarrow \mathrm{Z}$ which satisfies

(i) $\{u, v\}=-\{v, u\}$,

(ii) $\{u v, w\}+\{w u, v\}+\{v w, u\}=0$.

To prove the proposition, we prepare some notation. Let

$$
0 \longrightarrow \mathrm{Z} \longrightarrow \mathfrak{u} \stackrel{\pi}{\longrightarrow} \mathfrak{a} \longrightarrow 0,
$$

be a central extension. Notice that, for $x, y \in \mathfrak{a}$ and $x^{\prime}, y^{\prime} \in \mathfrak{u}$ such that $\pi\left(x^{\prime}\right)=x$ and $\pi\left(y^{\prime}\right)=y$, the commutator $\left[x^{\prime}, y^{\prime}\right]$ does not depend on the choice of the inverse images $x^{\prime}$ and $y^{\prime}$. Hence, in the sequel, we denote $\left[x^{\prime}, y^{\prime}\right]$ by $[x, y]^{\prime}$.

We first consider the case of $\mathfrak{s l}_{2}$ : Let us fix a basis of $\mathfrak{s l}_{2}$ as follows:

$$
\mathfrak{g}_{k}=k X_{+} \oplus k H \oplus k X_{-} \quad \text { such that }\left[X_{+}, X_{-}\right]=H,\left[H, X_{ \pm}\right]= \pm 2 X_{ \pm} .
$$

A non-degenerate even supersymmetric bilinear form on $\mathfrak{s l}_{2}$ is given by

$$
(H, H)=2, \quad\left(X_{+}, X_{-}\right)=1 .
$$

Lemma 4.12. For $a \in \mathrm{A}$, if we set

$$
\begin{aligned}
& \left(X_{ \pm} \otimes a\right)^{\prime}:= \pm \frac{1}{2}\left[H \otimes 1, X_{ \pm} \otimes a\right]^{\prime}, \\
& (H \otimes a)^{\prime}:=\left[X_{+} \otimes 1, X_{-} \otimes a\right]^{\prime},
\end{aligned}
$$


then there exists a pairing $\{\cdot, \cdot\}$ with the condition (23), and the following holds:

$$
\left[(X \otimes a)^{\prime},(Y \otimes b)^{\prime}\right]=([X, Y] \otimes a b)^{\prime}+(X, Y)\{a, b\},
$$

where $(\cdot, \cdot)$ is the above invariant form of $\mathfrak{g}_{k}, X, Y \in \mathfrak{g}_{k}$ and $a, b \in \mathrm{A}$.

Proof. Since $\pi\left(\left[(H \otimes a)^{\prime},(H \otimes b)^{\prime}\right]\right)=[H \otimes a, H \otimes b]=0$, we see that $[(H \otimes$ $\left.a)^{\prime},(H \otimes b)^{\prime}\right] \equiv 0(\bmod \mathrm{Z})$. Hence we set

$$
\{a, b\}:=\frac{1}{2}\left[(H \otimes a)^{\prime},(H \otimes b)^{\prime}\right] .
$$

To show this lemma, it suffices to show the following formulas:

F1. $\left[(H \otimes a)^{\prime},\left(X_{ \pm} \otimes b\right)^{\prime}\right]= \pm 2\left(X_{ \pm} \otimes a b\right)^{\prime}$

F2. $\left[\left(X_{+} \otimes a\right)^{\prime},\left(X_{-} \otimes b\right)^{\prime}\right]=(H \otimes a b)^{\prime}+\{a, b\}$, $\left[(H \otimes a)^{\prime},(H \otimes b)^{\prime}\right]=2\{a, b\}$,

F3. $\left[\left(X_{ \pm} \otimes a\right)^{\prime},\left(X_{ \pm} \otimes b\right)^{\prime}\right]=0$.

We prove $\mathbf{F} 1$. By definition we have

$$
\begin{aligned}
& {\left[(H \otimes a)^{\prime},\left(X_{ \pm} \otimes b\right)^{\prime}\right] } \\
= & \pm \frac{1}{2}\left[(H \otimes a)^{\prime},\left[(H \otimes 1)^{\prime},\left(X_{ \pm} \otimes b\right)^{\prime}\right]\right] \\
= & \pm \frac{1}{2}\left\{\left[\left[(H \otimes a)^{\prime},(H \otimes 1)^{\prime}\right],\left(X_{ \pm} \otimes b\right)^{\prime}\right]+\left[(H \otimes 1)^{\prime},\left[(H \otimes a)^{\prime},\left(X_{ \pm} \otimes b\right)^{\prime}\right]\right]\right\}
\end{aligned}
$$

Since

$$
\begin{aligned}
& {\left[(H \otimes a)^{\prime},(H \otimes 1)^{\prime}\right] \equiv 0(\bmod \mathrm{Z})} \\
& {\left[(H \otimes a)^{\prime},\left(X_{ \pm} \otimes b\right)^{\prime}\right]=\left[H \otimes a, X_{ \pm} \otimes b\right]^{\prime} \equiv \pm 2\left(X_{ \pm} \otimes a b\right)^{\prime}(\bmod \mathrm{Z}),}
\end{aligned}
$$

we have $\mathbf{F} 1$.

The second formula in F2 is nothing but the definition of $\{\cdot, \cdot\}$. By F1, we have

$$
\begin{aligned}
& {\left[\left(X_{+} \otimes a\right)^{\prime},\left(X_{-} \otimes b\right)^{\prime}\right] } \\
= & \frac{1}{2}\left[\left[(H \otimes a)^{\prime},\left(X_{+} \otimes 1\right)^{\prime}\right],\left(X_{-} \otimes b\right)^{\prime}\right] \\
= & \frac{1}{2}\left\{\left[\left[(H \otimes a)^{\prime},\left(X_{-} \otimes b\right)^{\prime}\right],\left(X_{+} \otimes 1\right)^{\prime}\right]+\left[(H \otimes a)^{\prime},\left[\left(X_{+} \otimes 1\right)^{\prime},\left(X_{-} \otimes b\right)^{\prime}\right]\right]\right\}
\end{aligned}
$$

Since by the definition of $(H \otimes b)^{\prime}$ and F1

$$
\begin{aligned}
& {\left[(H \otimes a)^{\prime},\left(X_{-} \otimes b\right)^{\prime}\right]=-2\left(X_{-} \otimes a b\right)^{\prime},} \\
& {\left[\left(X_{+} \otimes 1\right)^{\prime},\left(X_{-} \otimes b\right)^{\prime}\right]=(H \otimes b)^{\prime},}
\end{aligned}
$$

the first formula in $\mathbf{F} 2$ is proved.

We prove F3. Since $\left[\left(X_{ \pm} \otimes a\right)^{\prime},\left(X_{ \pm} \otimes b\right)^{\prime}\right] \equiv 0(\bmod \mathrm{Z})$, we have

$$
\begin{aligned}
0 & =\left[(H \otimes 1)^{\prime},\left[\left(X_{ \pm} \otimes a\right)^{\prime},\left(X_{ \pm} \otimes b\right)^{\prime}\right]\right] \\
& =\left[\left[(H \otimes 1)^{\prime},\left(X_{ \pm} \otimes a\right)^{\prime}\right],\left(X_{ \pm} \otimes b\right)^{\prime}\right]+\left[\left(X_{ \pm} \otimes a\right)^{\prime},\left[(H \otimes 1)^{\prime},\left(X_{ \pm} \otimes b\right)^{\prime}\right]\right] \\
& = \pm 4\left[\left(X_{ \pm} \otimes a\right)^{\prime},\left(X_{ \pm} \otimes b\right)^{\prime}\right] .
\end{aligned}
$$


Since $\frac{1}{2} \in k$, we obtain F3.

Finally let us check (23). (i) is clear from the definition of $\{\cdot, \cdot\}$. We prove (ii). By F1 and F2, we have

$$
\left[(H \otimes a)^{\prime},\left[\left(X_{+} \otimes b\right)^{\prime},\left(X_{-} \otimes c\right)^{\prime}\right]\right]=2\{a, b c\},
$$

and

$$
\begin{aligned}
& {\left[(H \otimes a)^{\prime},\left[\left(X_{+} \otimes b\right)^{\prime},\left(X_{-} \otimes c\right)^{\prime}\right]\right]} \\
& =\left[\left[(H \otimes a)^{\prime},\left(X_{+} \otimes b\right)^{\prime}\right],\left(X_{-} \otimes c\right)^{\prime}\right]+\left[\left(X_{+} \otimes b\right)^{\prime},\left[(H \otimes a)^{\prime},\left(X_{-} \otimes c\right)^{\prime}\right]\right] \\
& =2\{a b, c\}+2\{b, a c\} .
\end{aligned}
$$

Since $\frac{1}{2} \in k$, using (i) we obtain (ii).

Next we consider the case of $\mathfrak{o s p}(1,2)$ : Let us fix a basis of $\mathfrak{o s p}(1,2)$ as follows:

$$
\mathfrak{g}_{k}=\mathfrak{o s p}(1,2)=k X_{+} \oplus k x_{+} \oplus k H \oplus k x_{-} \oplus k X_{-},
$$

such that

$$
\begin{aligned}
{\left[H, X_{ \pm}\right] } & = \pm 4 X_{ \pm}, \quad\left[X_{+}, X_{-}\right]=\frac{1}{2} H, \quad X_{ \pm}= \pm \frac{1}{4}\left[x_{ \pm}, x_{ \pm}\right], \\
{\left[H, x_{ \pm}\right] } & = \pm 2 x_{ \pm}, \quad\left[x_{+}, x_{-}\right]=H, \quad\left[X_{ \pm}, x_{\mp}\right]=-x_{ \pm} .
\end{aligned}
$$

We remark that this gives the Chevalley basis for $\mathfrak{o s p}(1,2)$. A non-degenerate even supersymmetric bilinear form on $\mathfrak{o s p}(1,2)$ is given as follows:

$$
(H, H)=2, \quad\left(x_{+}, x_{-}\right)=1, \quad\left(X_{+}, X_{-}\right)=\frac{1}{4} .
$$

Lemma 4.13. For $a \in \mathrm{A}$, if we set

$$
\begin{aligned}
& \left(X_{ \pm} \otimes a\right)^{\prime}:= \pm \frac{1}{4}\left[H \otimes 1, X_{ \pm} \otimes a\right]^{\prime}, \\
& \left(x_{ \pm} \otimes a\right)^{\prime}:= \pm \frac{1}{2}\left[H \otimes 1, x_{ \pm} \otimes a\right]^{\prime}, \\
& (H \otimes a)^{\prime}:=\left[x_{+} \otimes 1, x_{-} \otimes a\right]^{\prime},
\end{aligned}
$$

then there exists a pairing $\{\cdot, \cdot\}$ such that

$$
\left[(X \otimes a)^{\prime},(Y \otimes b)^{\prime}\right]=([X, Y] \otimes a b)^{\prime}+(X, Y)\{a, b\},
$$

where $(\cdot, \cdot)$ is the above invariant form of $\mathfrak{g}_{k}, X, Y \in \mathfrak{g}_{k}$ and $a, b \in \mathrm{A}$.

Proof. Similarly to $\mathfrak{s l}_{2}$ case, we see that $\left[(H \otimes a)^{\prime},(H \otimes b)^{\prime}\right] \equiv 0(\bmod \mathrm{Z})$. Hence we set

$$
\{a, b\}:=\frac{1}{2}\left[(H \otimes a)^{\prime},(H \otimes b)^{\prime}\right] .
$$

To show this lemma, it suffices to show the following formulas:

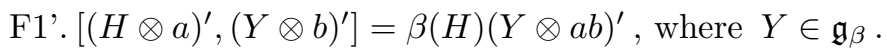


$\mathrm{F} 2 ' \cdot\left[\left(x_{+} \otimes a\right)^{\prime},\left(x_{-} \otimes b\right)^{\prime}\right]=(H \otimes a b)^{\prime}+\{a, b\}$, $\left[(H \otimes a)^{\prime},(H \otimes b)^{\prime}\right]=2\{a, b\}$.

F3'. $\left[\left(x_{ \pm} \otimes a\right)^{\prime},\left(x_{ \pm} \otimes b\right)^{\prime}\right]= \pm 4\left(X_{ \pm} \otimes a b\right)^{\prime}$.

$\mathrm{F} 4^{\prime} .\left[\left(X_{ \pm} \otimes a\right)^{\prime},\left(x_{\mp} \otimes b\right)^{\prime}\right]=-\left(x_{ \pm} \otimes a b\right)^{\prime}$.

F5'. $\left[\left(X_{+} \otimes a\right)^{\prime},\left(X_{-} \otimes b\right)^{\prime}\right]=\frac{1}{2}(H \otimes a b)^{\prime}+\frac{1}{4}\{a, b\}$.

$\mathrm{F} 6$ ' $\left[\left(X_{ \pm} \otimes a\right)^{\prime},\left(x_{ \pm} \otimes b\right)^{\prime}\right]=0$,

$\left[\left(X_{ \pm} \otimes a\right)^{\prime},\left(X_{ \pm} \otimes b\right)^{\prime}\right]=0$.

The proofs of $\mathbf{F} 1$ ' and $\mathbf{F} 2$ ' are similar to those of $\mathbf{F} 1$ and $\mathbf{F} 2$ in the $\mathfrak{s l}_{2}$-case respectively.

We prove F3'. Notice that $\left[\left(x_{ \pm} \otimes a\right)^{\prime},\left(x_{ \pm} \otimes b\right)^{\prime}\right] \equiv \pm 4\left(X_{ \pm} \otimes a b\right)^{\prime}(\bmod Z)$. Hence we have

$$
\left[(H \otimes 1)^{\prime},\left[\left(x_{ \pm} \otimes a\right)^{\prime},\left(x_{ \pm} \otimes b\right)^{\prime}\right]\right]= \pm 4\left[(H \otimes 1)^{\prime},\left(X_{ \pm} \otimes a b\right)^{\prime}\right]=16\left(X_{ \pm} \otimes a b\right)^{\prime} .
$$

On the other hand, by the Jacobi identity,

$$
\begin{aligned}
& {\left[(H \otimes 1)^{\prime},\left[\left(x_{ \pm} \otimes a\right)^{\prime},\left(x_{ \pm} \otimes b\right)^{\prime}\right]\right] } \\
= & {\left[\left[(H \otimes 1)^{\prime},\left(x_{ \pm} \otimes a\right)^{\prime}\right],\left(x_{ \pm} \otimes b\right)^{\prime}\right]+\left[\left(x_{ \pm} \otimes a\right)^{\prime},\left[(H \otimes 1)^{\prime},\left(x_{ \pm} \otimes b\right)^{\prime}\right]\right] } \\
= & \pm 4\left[\left(x_{ \pm} \otimes a\right)^{\prime},\left(x_{ \pm} \otimes b\right)^{\prime}\right] .
\end{aligned}
$$

Hence the condition $\frac{1}{2} \in k$ ensures $\mathbf{F} 3$ '.

The proof of $\mathbf{F} 4{ }^{\prime}$ is similar to that of F3'.

We prove F5'. By F2', F3' and F4', we have

$$
\begin{aligned}
& {\left[\left(X_{+} \otimes a\right)^{\prime},\left(X_{-} \otimes b\right)^{\prime}\right] } \\
= & \frac{1}{4}\left[\left[\left(x_{+} \otimes 1\right)^{\prime},\left(x_{+} \otimes a\right)^{\prime}\right],\left(X_{-} \otimes b\right)^{\prime}\right] \\
= & \frac{1}{4}\left\{\left[\left[\left(x_{+} \otimes 1\right)^{\prime},\left(X_{-} \otimes b\right)^{\prime}\right],\left(x_{+} \otimes a\right)^{\prime}\right]+\left[\left(x_{+} \otimes 1\right)^{\prime},\left[\left(x_{+} \otimes a\right)^{\prime},\left(X_{-} \otimes b\right)^{\prime}\right]\right]\right\} .
\end{aligned}
$$

Since

$$
\begin{aligned}
& {\left[\left(x_{+} \otimes 1\right)^{\prime},\left(X_{-} \otimes b\right)^{\prime}\right]=\left(x_{-} \otimes b\right)^{\prime},} \\
& {\left[\left(x_{+} \otimes a\right)^{\prime},\left(X_{-} \otimes b\right)^{\prime}\right]=\left(x_{-} \otimes a b\right)^{\prime},}
\end{aligned}
$$

by $\mathbf{F} 4$ ', we obtain

$$
\begin{aligned}
& {\left[\left(X_{+} \otimes a\right)^{\prime},\left(X_{-} \otimes b\right)^{\prime}\right] } \\
= & \frac{1}{4}\left\{\left[\left(x_{-} \otimes b\right)^{\prime},\left(x_{+} \otimes a\right)^{\prime}\right]+\left[\left(x_{+} \otimes 1\right)^{\prime},\left(x_{-} \otimes a b\right)^{\prime}\right]\right\} \\
= & \frac{1}{4}\left\{(H \otimes a b)^{\prime}+\{a, b\}+(H \otimes a b)^{\prime}+\{1, a b\}\right\} .
\end{aligned}
$$

Here we notice that if we take $u=a b, v=w=1$ in (23), then $\{1, a b\}=0$. Hence we have the conclusion.

By using the condition $\frac{1}{2}, \frac{1}{3} \in k$, we can prove $\mathbf{F 6}^{\prime}$ in a way similar to $\mathbf{F} 3$ in the $\mathfrak{s l}_{2}$-case. 
To show Theorem 4.7, we need the following preliminary lemma.

Lemma 4.14. Let $\Delta$ be the root system of a basic classical Lie superalgebra $\mathfrak{g}$. 1. If $\alpha \in \Delta_{\overline{1}}^{+}$such that $(\alpha, \alpha)=0$, then there exists $\gamma \in \Delta$ such that $\alpha\left(H_{\gamma}\right)=$ 1 .

2. If $\alpha, \beta \in \Delta$ and $\alpha+\beta \neq 0$, then there exists $\delta \in \Delta$ such that $(\alpha+\beta)\left(H_{\delta}\right)^{-1} \in$ $k$, where we exclude the case $\tilde{\alpha}+\tilde{\beta}= \pm\left(\tilde{\alpha}_{1}+2 \tilde{\alpha}_{2}+\tilde{\alpha}_{3}\right)$ for $\mathrm{A}(1,1)$ (see Remark 2.13 in $\S 2.3$ ).

Proof OF 1. By Lemma 2.22, we may assume that $\alpha=\alpha_{i_{0}}$. We see that if the Dynkin diagram contains the following type subdiagrams

$$
\alpha_{\lambda}^{\alpha_{j}} \alpha_{i_{0}}
$$

then we may choose $\gamma:=-\alpha_{j}$. Note that if the Dynkin diagram of $\mathfrak{g}$ does not contain the above type subdiagrams, then $\mathfrak{g}$ is of type $\mathrm{B}(0, n)$ or $\mathrm{B}(1,1)$, and further $\mathrm{B}(0, n)$ does not have a root of length 0 . In the case of $\mathrm{B}(1,1)$, we can easily check the lemma.

Proof of 2. We first consider the case where $\alpha$ and $\beta$ are linearly dependent. In this case, we distinguish the following cases:

Case1. $(\alpha, \alpha) \neq 0$.

Case2 . $(\alpha, \alpha)=0$.

For Case 1, $\beta= \pm 2 \alpha, \alpha, \pm \frac{1}{2} \alpha$, Lemma 2.23 says that if we choose $\delta:=\alpha$, then $(\alpha+\beta)\left(H_{\delta}\right)^{-1} \in k$. For Case2 , the first assertion implies that there exists $\gamma \in \Delta$ such that $\alpha\left(H_{\gamma}\right)=1$. Since $\beta=\alpha$, we may choose $\delta:=\gamma$.

Next we consider the case where $\alpha$ and $\beta$ are linearly independent. Let us put $\bar{\Delta}:=\Delta \cap(\mathbb{Z} \alpha \oplus \mathbb{Z} \beta)$. In this case, we distinguish the following subcases:

Case1. $\bar{\Delta}$ : a rank two system,

Case2 . $\bar{\Delta}$ : the disjoint union of two rank one systems.

In Case1, we can choose the simple roots of $\bar{\Delta}$ such that $\alpha$ is one of the simple roots and $\beta$ become a positive root. Then $\bar{\Delta}$ is of type $\mathrm{A}_{2}, \mathrm{~B}_{2}, \mathrm{G}_{2}$ or one of the rank two root systems listed in the proof of Lemma 3.5. For each case, the value of $\lambda$ is given as in Remark 3.6. Now the lemma follows from case by case checking.

In Case2, we also consider the following subcases:

Case2.1. $(\alpha, \alpha) \neq 0$ or $(\beta, \beta) \neq 0$.

Case2.2. $(\alpha, \alpha)=0$ and $(\beta, \beta)=0$.

In Case2.1, we may assume that $(\alpha, \alpha) \neq 0$ without loss of generality. Since $(\alpha, \beta)=0$ in this case, by Lemma 2.23 , if we take $\delta:=\alpha$, then the lemma holds.

Finally we prove the lemma in Case2.2 . By Lemma 2.22, we may assume that $\alpha=\alpha_{i_{0}}$. Note that $\left(\beta, \alpha_{i_{0}}\right)=0$ in this case. Since $\beta \in \Delta_{\overline{1}}$ satisfies $(\beta, \beta)=0$, 
$k \alpha_{i_{0}} \neq k \beta$ and $\left(\beta, \alpha_{i_{0}}\right)=0$, from the data of the root systems in Appendix $\mathrm{A}$, the cases where $\mathfrak{g}$ is of type $\mathrm{A}(m, 0), \mathrm{A}(0, n), \mathrm{B}(0 . n), \mathrm{B}(1, n), \mathrm{B}(m, 1)$, $\mathrm{C}(n), \mathrm{D}(m, 1), \mathrm{D}(2,1 ; a), \mathrm{F}(4)$ and $\mathrm{G}(3)$ are excluded. Hence we have to check the cases where $\mathfrak{g}$ is of type $\mathrm{A}(m, n)(m, n \geq 1)$ and $\mathrm{B}(m, n), \mathrm{D}(m, n)$ $(m, n \geq 2)$. In these cases, if there exists $j \in\left\{i_{0} \pm 1\right\}$ such that $\beta\left(H_{\alpha_{j}}\right)=0$, then $\left(\alpha_{i_{0}}+\beta\right)\left(H_{\alpha_{j}}\right) \in\{ \pm 1\}$ and thus we may choose $\delta:=\alpha_{j}$. For the rest of the case, i.e., the case when $\beta\left(H_{\alpha_{i_{0} \pm 1}}\right) \neq 0$ are given by the following table:

\begin{tabular}{|c|c|c|c|}
\hline type of $\mathfrak{g}$ & $\alpha_{i_{0}}$ & $\beta$ & $\delta$ \\
\hline $\mathrm{A}(1,1)$ & $\alpha_{2}$ & $\epsilon_{1}-\delta_{2}$ & $\times$ \\
\hline $\mathrm{A}(m, n)\left\{\begin{array}{l}(m, n \geq 1) \\
(m+n \geq 3)\end{array}\right.$ & $\alpha_{m+1}$ & $\epsilon_{m}-\delta_{2}$ & $\begin{cases}\alpha_{m-1} & (m \geq 2) \\
\alpha_{m+3} & (n \geq 2)\end{cases}$ \\
\cline { 3 - 5 } & & $\begin{array}{l}\epsilon_{m+1}-\delta_{2} \\
\epsilon_{m}-\delta_{1}\end{array}$ & $\alpha_{m+1}$ \\
\hline \multirow{2}{*}{$\begin{array}{l}\mathrm{B}(m, n) \quad(m, n \geq 2) \\
\mathrm{D}(m, n)\end{array}$} & $\alpha_{n}$ & $\delta_{n-1}-\epsilon_{2}$ & $\alpha_{n+2}$ \\
\cline { 3 - 5 } & & $\begin{array}{l}\delta_{n-1}+\epsilon_{2} \\
\delta_{n} \pm \epsilon_{2} \\
\delta_{n-1} \pm \epsilon_{1}\end{array}$ & $\alpha_{n+1}$ \\
\hline
\end{tabular}

The first case is excluded in this lemma, and for the other cases, we may choose $\delta$ as in the table.

Remark 2.19 implies that a non-degenerate even supersymmetric invariant bilinear form of $\mathfrak{g}_{k}$ is induced from an invariant form of $\overline{\mathfrak{g}}_{k}$. Hence to prove our main theorem (Theorem 4.7), we show the following proposition:

Proposition 4.15. Suppose that $\mathfrak{g}$ is a basic classical Lie superalgebras which is not of type $\mathrm{A}(1,1)$. Let $\mathrm{Z}$ be a free $k$-module and

$$
0 \longrightarrow \mathrm{Z} \longrightarrow \mathfrak{g}_{k}^{\prime}(\mathrm{A}) \stackrel{\pi}{\longrightarrow} \mathfrak{g}_{k} \otimes_{k} \mathrm{~A} \longrightarrow 0
$$

be a central extension of $\mathfrak{g}_{k} \otimes_{k} \mathrm{~A}$. Then the bracket of $\mathfrak{g}_{k}^{\prime}(\mathrm{A})$ can be described as

$$
\left[(X \otimes a)^{\prime},(Y \otimes b)^{\prime}\right]=([X, Y] \otimes a b)^{\prime}+(X, Y)\{a, b\},
$$

where $(\cdot, \cdot)$ is a non-degenerate even supersymmetric invariant bilinear form on $\mathfrak{g}_{k}$, and $\{\cdot, \cdot\}: \mathrm{A} \times \mathrm{A} \rightarrow \mathrm{Z}$ satisfies the condition (23).

Proof. Let $\left\{X_{\alpha}, H_{\alpha} \mid \alpha \in \Delta\right\}$ be a Chevalley basis as in Section 3. For $\alpha \in \Delta_{\overline{0}}^{+}$ such that $\frac{1}{2} \alpha \notin \Delta$, we set

$$
\begin{aligned}
& \left(X_{\beta} \otimes a\right)^{\prime}:=\frac{1}{\beta\left(H_{\alpha}\right)}\left[H_{\alpha} \otimes 1, X_{\beta} \otimes a\right]^{\prime}, \\
& \left(H_{\beta} \otimes a\right)^{\prime}:=\sigma_{\beta}\left[X_{\beta} \otimes 1, X_{-\beta} \otimes a\right]^{\prime},
\end{aligned}
$$


$(\beta= \pm \alpha)$ and

$$
\begin{aligned}
\tilde{\mathfrak{g}}(\alpha) & :=\left\{k X_{\alpha} \oplus k H_{\alpha} \oplus k X_{-\alpha}\right\} \otimes_{k} \mathrm{~A}, \\
\tilde{\mathfrak{g}}^{\prime}(\alpha) & :=\pi^{-1}(\tilde{\mathfrak{g}}(\alpha)) .
\end{aligned}
$$

For $\alpha \in \Delta_{\overline{1}}^{+}$such that $2 \alpha \in \Delta$, we set

$$
\begin{aligned}
& \left(X_{\beta} \otimes a\right)^{\prime}:=\frac{1}{\beta\left(H_{\alpha}\right)}\left[H_{\alpha} \otimes 1, X_{\beta} \otimes a\right]^{\prime}, \\
& \left(H_{\beta} \otimes a\right)^{\prime}:=\sigma_{\beta}\left[X_{\beta} \otimes 1, X_{-\beta} \otimes a\right]^{\prime},
\end{aligned}
$$

$(\beta= \pm \alpha, \pm 2 \alpha)$ and

$$
\begin{aligned}
& \tilde{\mathfrak{g}}(\alpha):=\left\{k X_{2 \alpha} \oplus k X_{\alpha} \oplus k H_{\alpha} \oplus k X_{-\alpha} \oplus k X_{-2 \alpha}\right\} \otimes_{k} \mathrm{~A}, \\
& \tilde{\mathfrak{g}}^{\prime}(\alpha):=\pi^{-1}(\tilde{\mathfrak{g}}(\alpha)) .
\end{aligned}
$$

Notice that from Proposition 4.11, we see that, for $\alpha \in \Delta$ such that $(\alpha, \alpha) \neq 0$, there exists $\{\cdot, \cdot\}_{\alpha}: \mathrm{A} \times \mathrm{A} \rightarrow \mathrm{Z}$ such that

$$
\left[(X \otimes a)^{\prime},(Y \otimes b)^{\prime}\right]=([X, Y] \otimes a b)^{\prime}+(X, Y)\{a, b\}_{\alpha}
$$

holds for any $(X \otimes a)^{\prime}$ and $(Y \otimes b)^{\prime} \in \tilde{\mathfrak{g}}^{\prime}(\alpha)$.

For each $\alpha \in \Delta_{\overline{1}}$ such that $2 \alpha \notin \Delta$ i.e. $(\alpha, \alpha)=0$, we fix $\gamma \in \Delta$ as in Lemma 4.14, and set

$$
\begin{aligned}
& \left(X_{\beta} \otimes a\right)^{\prime}:=\frac{1}{\beta\left(H_{\gamma}\right)}\left[H_{\gamma} \otimes 1, X_{\beta} \otimes a\right]^{\prime}, \\
& \left(H_{\beta} \otimes a\right)^{\prime}:=\sigma_{\beta}\left[X_{\beta} \otimes 1, X_{-\beta} \otimes a\right]^{\prime}
\end{aligned}
$$

$(\beta:= \pm \alpha)$.

For simplicity, we introduce the following notation:

$$
\gamma(\alpha):= \begin{cases}\alpha & \text { if } \quad(\alpha, \alpha) \neq 0 \\ \gamma & \text { if }(\alpha, \alpha)=0\end{cases}
$$

where $\gamma$ is taken as above. From now on, we will divided the proof of Proposition 4.15 to several steps (Lemma $4.16-4.21$ ).

Lemma 4.16. For $\alpha, \beta \in \Delta$, we have

$$
\left[\left(H_{\alpha} \otimes a\right)^{\prime},\left(X_{\beta} \otimes b\right)^{\prime}\right]=\beta\left(H_{\alpha}\right)\left(X_{\beta} \otimes a b\right)^{\prime} .
$$

Proof. We can prove this lemma in a way similar to $\mathbf{F} 1$ in Lemma 4.12.

Lemma 4.17. For each $\alpha \in \Delta$, we set $\gamma:=\gamma(\alpha)$ and

$$
\{a, b\}_{\alpha}:=\left[\left(H_{\gamma} \otimes a\right)^{\prime},\left(H_{\alpha} \otimes b\right)^{\prime}\right], \quad\{a, b\}:=\frac{\sigma_{\alpha}}{\left(X_{\alpha}, X_{-\alpha}\right)}\{a, b\}_{\alpha},
$$


where $\sigma_{\alpha}$ is defined as in (11). Then $\{\cdot, \cdot\}$ is independent of the choice of $\alpha$ and

$$
\begin{aligned}
& {\left[\left(X_{\alpha} \otimes a\right)^{\prime},\left(X_{-\alpha} \otimes b\right)^{\prime}\right]=\sigma_{\alpha}\left(H_{\alpha} \otimes a b\right)^{\prime}+\left(X_{\alpha}, X_{-\alpha}\right)\{a, b\},} \\
& {\left[\left(H_{\alpha} \otimes a\right)^{\prime},\left(H_{\beta} \otimes b\right)^{\prime}\right]=\left(H_{\alpha}, H_{\beta}\right)\{a, b\} .}
\end{aligned}
$$

Proof. By Lemma 4.16 we have

$$
\begin{aligned}
& {\left[\left(X_{\alpha} \otimes a\right)^{\prime},\left(X_{-\alpha} \otimes b\right)^{\prime}\right]} \\
& =\left[\left[\left(H_{\gamma} \otimes a\right)^{\prime},\left(X_{\alpha} \otimes 1\right)^{\prime}\right],\left(X_{-\alpha} \otimes b\right)^{\prime}\right] \\
& =\left[\left(H_{\gamma} \otimes a\right)^{\prime},\left[\left(X_{\alpha} \otimes 1\right)^{\prime},\left(X_{-\alpha} \otimes b\right)^{\prime}\right]\right]-\left[\left(X_{\alpha} \otimes 1\right)^{\prime},\left[\left(H_{\gamma} \otimes a\right)^{\prime},\left(X_{-\alpha} \otimes b\right)^{\prime}\right]\right] \\
& =\sigma_{\alpha}\left[\left(H_{\gamma} \otimes a\right)^{\prime},\left(H_{\alpha} \otimes b\right)^{\prime}\right]+\left[\left(X_{\alpha} \otimes 1\right)^{\prime},\left(X_{-\alpha} \otimes a b\right)^{\prime}\right] \\
& =\sigma_{\alpha}\left(H_{\alpha} \otimes a b\right)^{\prime}+\sigma_{\alpha}\{a, b\}_{\alpha} .
\end{aligned}
$$

On the other hand,

$$
\begin{aligned}
& {\left[\left(H_{\alpha} \otimes a\right)^{\prime},\left(H_{\beta} \otimes b\right)^{\prime}\right]} \\
& =\sigma_{\beta}\left[\left(H_{\alpha} \otimes a\right)^{\prime},\left[\left(X_{\beta} \otimes 1\right)^{\prime},\left(X_{-\beta} \otimes b\right)^{\prime}\right]\right] \\
& =\sigma_{\beta}\left\{\left[\left[\left(H_{\alpha} \otimes a\right)^{\prime},\left(X_{\beta} \otimes 1\right)^{\prime}\right],\left(X_{-\beta} \otimes b\right)^{\prime}\right]+\left[\left(X_{\beta} \otimes 1\right)^{\prime},\left[\left(H_{\alpha} \otimes a\right)^{\prime},\left(X_{-\beta} \otimes b\right)^{\prime}\right]\right\}\right. \\
& =\beta\left(H_{\alpha}\right)\left\{\left(H_{\beta} \otimes a b\right)^{\prime}+\{a, b\}_{\beta}-\left(H_{\beta} \otimes a b\right)^{\prime}-\{1, a b\}_{\beta}\right\} \\
& =\beta\left(H_{\alpha}\right)\{a, b\}_{\beta} .
\end{aligned}
$$

Similarly, we obtain

$$
\begin{aligned}
& {\left[\left(H_{\alpha} \otimes a\right)^{\prime},\left(H_{\beta} \otimes b\right)^{\prime}\right]} \\
& =\left[\left[\left(X_{\alpha} \otimes 1\right)^{\prime},\left(X_{-\alpha} \otimes a\right)^{\prime}\right],\left(H_{\beta} \otimes b\right)^{\prime}\right] \\
& =\alpha\left(H_{\beta}\right)\{a, b\}_{\alpha} .
\end{aligned}
$$

Hence

$$
\beta\left(H_{\alpha}\right)\{a, b\}_{\beta}=\alpha\left(H_{\beta}\right)\{a, b\}_{\alpha} .
$$

Since

$$
\left(H_{\alpha}, H_{\beta}\right)=\sigma_{\beta}\left(H_{\alpha},\left[X_{\beta}, X_{-\beta}\right]\right)=\sigma_{\beta} \beta\left(H_{\alpha}\right)\left(X_{\beta}, X_{-\beta}\right)=\sigma_{\alpha} \alpha\left(H_{\beta}\right)\left(X_{\alpha}, X_{-\alpha}\right),
$$

we see that

$$
\{a, b\}:=\frac{\sigma_{\alpha}}{\left(X_{\alpha}, X_{-\alpha}\right)}\{a, b\}_{\alpha}
$$

is independent of the choice of $\alpha$. Moreover, we have

$$
\left[\left(H_{\alpha} \otimes a\right)^{\prime},\left(H_{\beta} \otimes b\right)^{\prime}\right]=\left(H_{\alpha}, H_{\beta}\right)\{a, b\}
$$

and

$$
\left[\left(X_{\alpha} \otimes a\right)^{\prime},\left(X_{-\alpha} \otimes b\right)^{\prime}\right]=\sigma_{\alpha}\left(H_{\alpha} \otimes a b\right)^{\prime}+\left(X_{\alpha}, X_{-\alpha}\right)\{a, b\}
$$


Now we complete the proof.

Lemma 4.18. $\alpha, \beta \in \Delta$ such that $\alpha+\beta \neq 0$.

$$
\left[\left(X_{\alpha} \otimes a\right)^{\prime},\left(X_{\beta} \otimes b\right)^{\prime}\right]=N_{\alpha, \beta}\left(X_{\alpha+\beta} \otimes a b\right)^{\prime},
$$

where $N_{\alpha, \beta}$ are defined as in (13).

Proof. For $\alpha, \beta \in \Delta$, we take $\delta \in \Delta$ as in Lemma 4.14. Notice that

$$
\left[\left(X_{\alpha} \otimes a\right)^{\prime},\left(X_{\beta} \otimes b\right)^{\prime}\right] \equiv N_{\alpha, \beta}\left(X_{\alpha+\beta} \otimes a b\right)^{\prime}(\bmod \mathrm{Z}) .
$$

Thus we have

$$
\left[\left(H_{\delta} \otimes 1\right)^{\prime},\left[\left(X_{\alpha} \otimes a\right)^{\prime},\left(X_{\beta} \otimes b\right)^{\prime}\right]\right]=N_{\alpha, \beta}(\alpha+\beta)\left(H_{\delta}\right)\left(X_{\alpha+\beta} \otimes a b\right)^{\prime},
$$

and

$$
\begin{aligned}
& {\left[\left(H_{\delta} \otimes 1\right)^{\prime},\left[\left(X_{\alpha} \otimes a\right)^{\prime},\left(X_{\beta} \otimes b\right)^{\prime}\right]\right]} \\
& =\left[\left[\left(H_{\delta} \otimes 1\right)^{\prime},\left(X_{\alpha} \otimes a\right)^{\prime}\right],\left(X_{\beta} \otimes b\right)^{\prime}\right]+\left[\left(X_{\alpha} \otimes a\right)^{\prime},\left[\left(H_{\delta} \otimes 1\right)^{\prime},\left(X_{\beta} \otimes b\right)^{\prime}\right]\right] \\
& =(\alpha+\beta)\left(H_{\delta}\right)\left[\left(X_{\alpha} \otimes a\right)^{\prime},\left(X_{\beta} \otimes b\right)^{\prime}\right] .
\end{aligned}
$$

Since $\left\{(\alpha+\beta)\left(H_{\delta}\right)\right\}^{-1} \in k$, we obtain the conclusion.

Lemma 4.19. For $\alpha \in \Delta$,

$$
\left(H_{-\alpha} \otimes a\right)^{\prime}=-\left(H_{\alpha} \otimes a\right)^{\prime} .
$$

Proof. We may assume that $\alpha \in \Delta^{+}$. By Lemma 4.17, we have

$$
\begin{aligned}
\left(H_{-\alpha} \otimes a\right)^{\prime} & =\sigma_{-\alpha}\left[\left(X_{-\alpha} \otimes 1\right)^{\prime},\left(X_{\alpha} \otimes a\right)^{\prime}\right] \\
& =-(-1)^{|\alpha|} \sigma_{-\alpha}\left[\left(X_{\alpha} \otimes a\right)^{\prime},\left(X_{-\alpha} \otimes 1\right)^{\prime}\right] \\
& =-\left\{\left(H_{\alpha} \otimes a\right)^{\prime}+\{a, 1\}_{\alpha}\right\} \\
& =-\left(H_{\alpha} \otimes a\right)^{\prime} .
\end{aligned}
$$

Lemma 4.20. For $\alpha, \beta \in \Delta$ such that $\alpha+\beta \in \Delta$, we have

$$
\left(H_{\alpha+\beta} \otimes a\right)^{\prime}=\frac{\varepsilon_{\alpha+\beta}}{\varepsilon_{\alpha}}\left(H_{\alpha} \otimes a\right)^{\prime}+\frac{\varepsilon_{\alpha+\beta}}{\varepsilon_{\beta}}\left(H_{\beta} \otimes a\right)^{\prime} .
$$


Proof. By Lemma 4.18, we have

$$
\begin{aligned}
& N_{-\alpha,-\beta} \sigma_{-\alpha-\beta}\left(H_{-\alpha-\beta} \otimes a\right)^{\prime} \\
& =\left[\left[\left(X_{-\alpha} \otimes 1\right)^{\prime},\left(X_{-\beta} \otimes 1\right)^{\prime}\right],\left(X_{\alpha+\beta} \otimes a\right)^{\prime}\right] \\
& =\left[\left(X_{-\alpha} \otimes 1\right)^{\prime},\left[\left(X_{-\beta} \otimes 1\right)^{\prime},\left(X_{\alpha+\beta} \otimes a\right)^{\prime}\right]\right] \\
& \quad \quad+(-1)^{|\beta||\alpha+\beta|}\left[\left[\left(X_{-\alpha} \otimes 1\right)^{\prime},\left(X_{\alpha+\beta} \otimes a\right)^{\prime}\right],\left(X_{-\beta} \otimes 1\right)^{\prime}\right] \\
& =N_{-\beta, \alpha+\beta} \sigma_{-\alpha}\left(H_{-\alpha} \otimes a\right)^{\prime}+(-1)^{|\beta||\alpha+\beta|} N_{-\alpha, \alpha+\beta} \sigma_{\beta}\left(H_{\beta} \otimes a\right)^{\prime} .
\end{aligned}
$$

Here we notice that, from Theorem 3.9 and the data of the root systems in Appendix A, we see that $N_{\alpha, \beta}^{-1} \in k$ for any $\alpha, \beta \in \Delta$. Hence by using (11), the definition of $N_{\alpha, \beta}$ in (13), Lemma 3.3 and Lemma 4.19, the lemma is proved.

Lemma 4.21. $\{\cdot, \cdot\}: \mathrm{A} \times \mathrm{A} \rightarrow \mathrm{Z}$ satisfies

(i) $\{u, v\}=-\{v, u\}$,

(ii) $\{u v, w\}+\{w u, v\}+\{v w, u\}=0$.

Proof. We show the first assertion. For $\alpha \in \Delta$ such that $\left(H_{\alpha}, H_{\alpha}\right) \neq 0$, we have

$$
\left[\left(H_{\alpha} \otimes a\right)^{\prime},\left(H_{\alpha} \otimes b\right)^{\prime}\right]=\left(H_{\alpha}, H_{\alpha}\right)\{a, b\} .
$$

Hence we have proved (i).

We prove (ii). For $\alpha \in \Delta$ such that $\alpha\left(H_{\alpha}\right) \neq 0$, by Lemma $4.16-4.20$, we have

$$
\left[\left(H_{\alpha} \otimes a\right)^{\prime},\left[\left(X_{\alpha} \otimes b\right)^{\prime},\left(X_{-\alpha} \otimes c\right)^{\prime}\right]\right]=\sigma_{\alpha}\left(H_{\alpha}, H_{\alpha}\right)\{a, b c\}
$$

and

$$
\begin{aligned}
& {\left[\left(H_{\alpha} \otimes a\right)^{\prime},\left[\left(X_{\alpha} \otimes b\right)^{\prime},\left(X_{-\alpha} \otimes c\right)^{\prime}\right]\right]} \\
& =\left[\left[\left(H_{\alpha} \otimes a\right)^{\prime},\left(X_{\alpha} \otimes b\right)^{\prime}\right],\left(X_{-\alpha} \otimes c\right)^{\prime}\right]+\left[\left(X_{\alpha} \otimes b\right)^{\prime},\left[\left(H_{\alpha} \otimes a\right)^{\prime},\left(X_{-\alpha} \otimes c\right)^{\prime}\right]\right] \\
& =\alpha\left(H_{\alpha}\right)\left(X_{\alpha}, X_{-\alpha}\right)\{a b, c\}+(-\alpha)\left(H_{\alpha}\right)\left(X_{\alpha}, X_{-\alpha}\right)\{b, a c\} .
\end{aligned}
$$

Since

$$
\sigma_{\alpha}\left(H_{\alpha}, H_{\alpha}\right)=\left(H_{\alpha},\left[X_{\alpha}, X_{-\alpha}\right]\right)=\left(\left[H_{\alpha}, X_{\alpha}\right], X_{-\alpha}\right)=\alpha\left(H_{\alpha}\right)\left(X_{\alpha}, X_{-\alpha}\right),
$$

using (i) we obtain (ii).

Remark 4.4.22. In the case where $\mathfrak{g}$ is of type $\mathrm{A}(1,1)$, Lemma 4.16, 4.17, 4.19 and 4.21 can be proved by the same argument as above. For Lemma 4.18 , it holds except for the case of $\alpha, \beta \in \Delta$ such that $\tilde{\alpha}+\tilde{\beta}= \pm\left(\tilde{\alpha}_{1}+2 \tilde{\alpha}_{2}+\tilde{\alpha}_{3}\right)$, since this cases are excluded in Lemma 4.14. Moreover, Lemma 4.20 holds, since $\pm\left(\tilde{\alpha}_{1}+2 \tilde{\alpha}_{2}+\tilde{\alpha}_{3}\right) \notin \tilde{\Delta}$. In the case of $\mathrm{A}(1,1)$, the following holds: 
Lemma 4.23. For $a, b, c, d \in \mathrm{A}$ such that $a b=c d$, we have

$$
\begin{aligned}
& {\left[\left(X_{ \pm\left(\alpha_{1}+\alpha_{2}\right)} \otimes a\right)^{\prime},\left(X_{ \pm\left(\alpha_{2}+\alpha_{3}\right)} \otimes b\right)^{\prime}\right]} \\
& =-\left[\left(X_{ \pm \alpha_{2}} \otimes c\right)^{\prime},\left(X_{ \pm\left(\alpha_{1}+\alpha_{2}+\alpha_{3}\right)} \otimes d\right)^{\prime}\right] .
\end{aligned}
$$

Proof. By using Lemma 4.18 for $\mathrm{A}(1,1)$, we have

$$
\begin{aligned}
& {\left[\left(X_{ \pm\left(\alpha_{1}+\alpha_{2}\right)} \otimes a\right)^{\prime},\left(X_{ \pm\left(\alpha_{2}+\alpha_{3}\right)} \otimes b\right)^{\prime}\right]} \\
& = \pm\left[\left(\left(X_{ \pm \alpha_{1}} \otimes a\right)^{\prime},\left(X_{ \pm \alpha_{2}} \otimes 1\right)^{\prime}\right],\left(X_{ \pm\left(\alpha_{2}+\alpha_{3}\right)} \otimes b\right)^{\prime}\right] \\
& =\mp\left[\left(\left(X_{ \pm \alpha_{1}} \otimes a\right)^{\prime},\left(X_{ \pm\left(\alpha_{2}+\alpha_{3}\right)} \otimes b\right)^{\prime}\right],\left(X_{ \pm \alpha_{2}} \otimes 1\right)^{\prime}\right] \\
& =-\left[\left(X_{ \pm\left(\alpha_{1}+\alpha_{2}+\alpha_{3}\right)} \otimes a b\right)^{\prime},\left(X_{ \pm \alpha_{2}} \otimes 1\right)^{\prime}\right] .
\end{aligned}
$$

On the other hand, from Lemma 4.16 we have

$$
\begin{aligned}
& {\left[\left(X_{ \pm \alpha_{2}} \otimes c\right)^{\prime},\left(X_{ \pm\left(\alpha_{1}+\alpha_{2}+\alpha_{3}\right)} \otimes d\right)^{\prime}\right]} \\
& =\mp\left[\left[\left(H_{1} \otimes c\right)^{\prime},\left(X_{ \pm \alpha_{2}} \otimes 1\right)^{\prime}\right],\left(X_{ \pm\left(\alpha_{1}+\alpha_{2}+\alpha_{3}\right)} \otimes d\right)^{\prime}\right] \\
& = \pm\left[\left(X_{ \pm \alpha_{2}} \otimes 1\right)^{\prime},\left[\left(H_{1} \times c\right)^{\prime},\left(X_{ \pm\left(\alpha_{1}+\alpha_{2}+\alpha_{3}\right)} \otimes d\right)^{\prime}\right]\right] \\
& =\left[\left(X_{ \pm\left(\alpha_{1}+\alpha_{2}+\alpha_{3}\right)} \otimes c d\right)^{\prime},\left(X_{ \pm \alpha_{2}} \otimes 1\right)^{\prime}\right] .
\end{aligned}
$$

Hence we have proved the lemma.

Before proving the main theorem, we consider the case where $\mathrm{A}=k$.

Proposition 4.24. The Lie superalgebra $\overline{\mathfrak{g}}_{k}$ is the universal central extension of $\mathfrak{g}_{k}$.

Proof. Let $0 \rightarrow \mathrm{Z} \rightarrow \mathfrak{a}_{k} \rightarrow \mathfrak{g}_{k} \rightarrow 0$ be the universal central extension of $\mathfrak{g}_{k}$. First we consider the case where $\mathfrak{g}$ is not of type $\mathrm{A}(n, n)$. By setting $\mathrm{A}:=k$ and $a, b:=1$ in Proposition 4.15 , we have $\mathfrak{a}_{k} \simeq \mathfrak{g}_{k}$. Hence we have proved the proposition in this case.

Next we consider the case where $\mathfrak{g}$ is of type $\mathrm{A}(n, n)$ for some $n \geq 2$. In this case, we have to check whether $\mathfrak{a}_{k}$ inherits the additional relation

$$
\sum_{i=1}^{n} i\left(h_{i}-h_{2 n+2-i}\right)-(n+1) h_{n+1}=0
$$

viz., we have to check two possibilities $\mathfrak{a}_{k} \simeq \mathfrak{g}_{k}$ or $\mathfrak{a}_{k} \simeq \overline{\mathfrak{g}}_{k} \simeq \mathfrak{s l}(n+1 \mid n+1)$. Since the short exact sequence (5) does not split, we conclude that $\mathfrak{a}_{k} \simeq \overline{\mathfrak{g}}_{k}$.

Finally, we consider the case where $\mathfrak{g}_{k}$ is of type $\mathrm{A}(1,1)$. Similarly to the above case, from Remark 4.22 and Lemma 4.23, we see that one of the Lie superalgebras $\mathfrak{g}_{k}, \mathfrak{s l}(2,2)$ and $\mathfrak{d}$ gives the universal central extension of $\mathfrak{g}_{k}$. Since the sequence (4) and (5) do not split, we obtain the conclusion.

Let us prove our main theorem (Theorem 4.7) by using Proposition 4.15. First we assume that $\mathfrak{g}$ is not of type $\mathrm{A}(n, n)$ for any $n$. Let $\phi$ be the map $\mathrm{A} \times \mathrm{A} \rightarrow$ 
$H \mathrm{C}_{2}(\mathrm{~A})$ defined by $(a, b) \mapsto a \otimes b$. Since the Lie superalgebra $\mathfrak{g}_{k} \otimes_{k} \mathrm{~A}$ is perfect, it has a universal central extension by Proposition 4.1. By the definition of $H_{2}(\mathrm{~A})$ and Proposition 4.15, for any central extension

$$
0 \longrightarrow \mathrm{Z} \longrightarrow \mathfrak{g}_{k}^{\prime}(\mathrm{A}) \longrightarrow \mathfrak{g}_{k} \otimes_{k} \mathrm{~A} \longrightarrow 0
$$

there exists $\psi: \mathrm{HC}_{2}(\mathrm{~A}) \rightarrow \mathrm{Z}$ such that the following diagram commutes

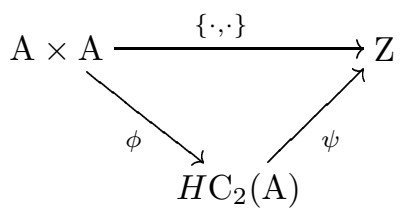

Hence the central extension

$$
0 \longrightarrow \mathrm{HC}_{2}(\mathrm{~A}) \longrightarrow \mathfrak{g}_{k}(\mathrm{~A}) \longrightarrow \mathfrak{g}_{k} \otimes_{k} \mathrm{~A} \longrightarrow 0
$$

satisfies the property of Definition $4.2,2$. Since $\phi$ is surjective, $\mathfrak{g}_{k}(\mathrm{~A})$ is perfect. Hence the above central extension is a universal central extension.

Next we consider the case of $\mathrm{A}(n, n)$. In this case, the above argument is insufficient, since there is a degeneration in $\mathfrak{h}$ as well as in $\mathfrak{h}^{*}$, i.e.,

$$
\begin{aligned}
& \sum_{i=1}^{n} i\left\{h_{i}-h_{2 n+2-i}\right\}-(n+1) h_{n+1}=0, \\
& \sum_{i=1}^{n} i\left(\alpha_{i}+\alpha_{2 n+2-i}\right)+(n+1) \alpha_{n+1}=0
\end{aligned}
$$

hold in $\mathfrak{g}_{k}$. To determine $\mathfrak{g}_{k}(\mathrm{~A})$, we consider the following commutative diagram:

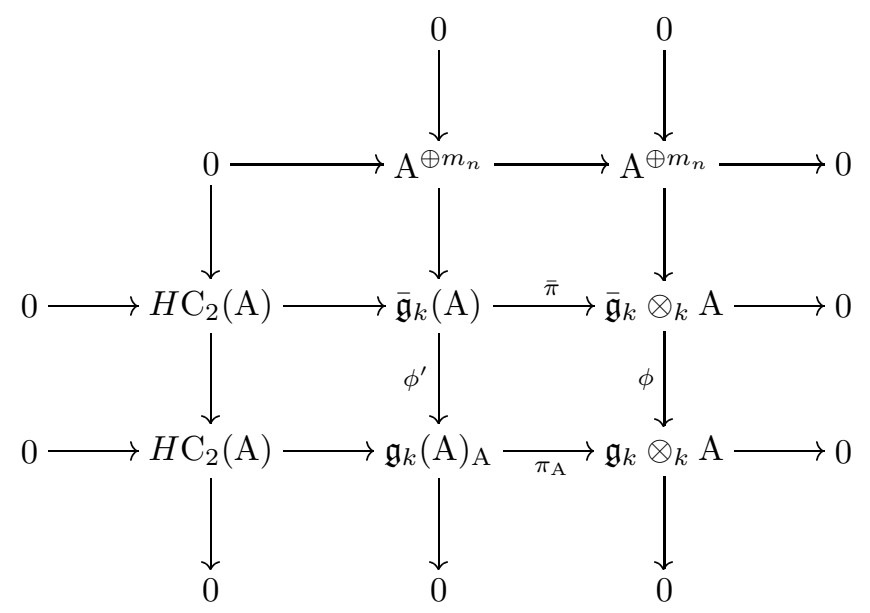


where the $m_{n}$ are defined by

$$
m_{n}:=\left\{\begin{array}{ll}
3 & \text { if } n=1 \\
1 & \text { if } n>1
\end{array} .\right.
$$

Note that each short sequence is exact. From this diagram, we obtain

$$
0 \longrightarrow \mathrm{HC}_{2}(\mathrm{~A}) \oplus \mathrm{A}^{\oplus m_{n}} \longrightarrow \overline{\mathfrak{g}}_{k}(\mathrm{~A}) \longrightarrow \mathfrak{g}_{k} \otimes_{k} \mathrm{~A} \longrightarrow 0 .
$$

Moreover, the sequence of the second row in (26) does not split, because if there exists $\iota^{\prime}: \overline{\mathfrak{g}}_{k}(\mathrm{~A}) \rightarrow \mathfrak{g}_{k}(\mathrm{~A})_{\mathrm{A}}$ such that $\tilde{\phi}^{\prime} \circ \iota^{\prime}=\mathrm{i} d$, then $\iota: \mathfrak{g}_{k} \otimes_{k} \mathrm{~A} \rightarrow \overline{\mathfrak{g}}_{k} \otimes_{k} \mathrm{~A}$ defined by $\iota(x):=\bar{\pi} \circ \iota^{\prime} \circ \pi_{\mathrm{A}}^{-1}(x) \quad\left(x \in \mathfrak{g}_{k} \otimes_{k} \mathrm{~A}\right)$ is well-defined and satisfies $\phi \circ \iota=\mathrm{i} d$. From the Lie superalgebra structures of $\overline{\mathfrak{g}}_{k}$ and $\mathfrak{g}_{k}$, we see that the sequence of the third row in (26) does not split. This is a contradiction and thus $\mathfrak{g}_{k}(\mathrm{~A})_{\mathrm{A}}$ is not the universal central extension.

On the other hand, it is easy to see that $\overline{\mathfrak{g}}_{k}(\mathrm{~A})$ is perfect and further it enjoys universality by Proposition 4.15. Now we can conclude that

$$
\overline{\mathfrak{g}}_{k}(\mathrm{~A}) \simeq \overline{\mathfrak{g}}_{k} \otimes_{k} \mathrm{~A} \oplus H \mathrm{C}_{2}(\mathrm{~A}) \quad(\text { as a } k \text {-module })
$$

gives the universal central extension of $\mathfrak{g}_{k} \otimes_{k} \mathrm{~A}$.

\section{A. Data of basic classical Lie superalgebras}

In this appendix, we will collect some data (e.g. Dynkin diagrams, Cartan matrix and root systems) of the basic classical Lie superalgebras.

Before giving these data, we explain how to recover the Cartan matrix from a given Dynkin diagram. Let us take one of the Dynkin diagrams listed below. We define the Cartan matrix $\mathrm{A}:=\left(a_{i, j}\right)$ associated with the diagram as follows: The set $\mathrm{I}$ is given by the index set of all vertices and $\tau$ is given by the subset corresponding to the vertices of type $\bigcirc$ and $\otimes$. The matrix elements $a_{i, j}$ are given by

$$
a_{i, i}:=\left\{\begin{array}{ll}
0 & \text { if } \alpha_{i} \text { corresponds to } \otimes \\
2 & \text { if } \alpha_{i} \text { corresponds to } \bigcirc \text { or }
\end{array},\right.
$$

and for $i \neq j$,

$$
a_{i, j}:=0
$$

if the vertices corresponding to $\alpha_{i}$ and $\alpha_{j}$ are not connected, and

$$
\begin{aligned}
a_{j, i} & := \begin{cases}\lambda & \text { if } \alpha_{j} \text { corresponds to } \otimes \\
-1 & \text { if } \alpha_{j} \text { corresponds to } \bigcirc \text { or }\end{cases} \\
a_{i, j} & :=\left\{\begin{array}{ll}
\lambda & \text { if } \alpha_{i} \text { corresponds to } \otimes \\
-k & \text { if } \alpha_{i} \text { corresponds to } \bigcirc \text { or }
\end{array},\right.
\end{aligned}
$$


for $i, j$ such that

$$
\stackrel{\alpha_{j}}{\stackrel{k \text {-fold }}{\longrightarrow}} \stackrel{\alpha_{i}}{\bigodot}
$$

where $\odot$ denotes $\bigcirc, \bigcirc$ or $\otimes$.

The following list contains a Dynkin diagram, a Cartan matrix, the set of the positive even or odd roots and simple roots. We remark that for a basic classical Lie superalgebra, the Dynkin diagram is not determined uniquely, if it contains a vertex of type $\otimes$. The Dynkin diagrams given here correspond to the choice of simple roots in the same list.

1. $\mathrm{A}(m, n)$ case:

$$
\begin{aligned}
& \alpha_{1} \quad \alpha_{m+1} \quad \alpha_{m+n+1} \\
& \bigcirc-\cdots \cdot-\overline{-}_{1} \otimes \frac{-1}{-1} \bigcirc-\cdots \cdot-\bigcirc
\end{aligned}
$$

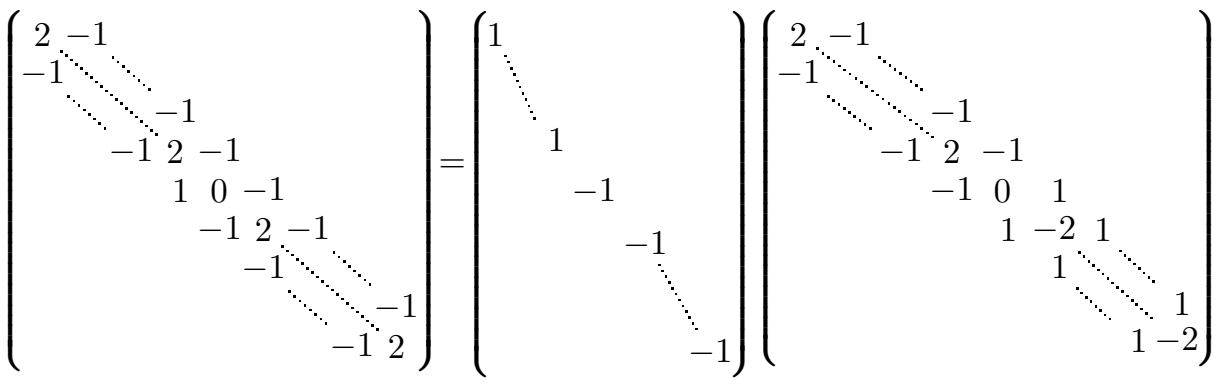

$$
\begin{aligned}
& \Delta_{\overline{0}}^{+}=\left\{\epsilon_{i}-\epsilon_{j}, \delta_{k}-\delta_{l} \mid 1 \leq i<j \leq m+1,1 \leq k<l \leq n+1\right\} . \\
& \Delta_{\overline{1}}^{+}=\left\{\epsilon_{i}-\delta_{j} \mid 1 \leq i \leq m+1,1 \leq j \leq n+1\right\} . \\
& \Pi=\left\{\epsilon_{1}-\epsilon_{2}, \cdots, \epsilon_{m}-\epsilon_{m+1}, \epsilon_{m+1}-\delta_{1}, \delta_{1}-\delta_{2}, \cdots, \delta_{n}-\delta_{n+1}\right\},
\end{aligned}
$$

where $\left\{\epsilon_{i}, \delta_{j} \mid 1 \leq i \leq m+1,1 \leq j \leq n+1\right\}$ is an orthogonal basis such that $\left(\epsilon_{i}, \epsilon_{i}\right)=1$ and $\left(\delta_{j}, \delta_{j}\right)=-1$.

2. $\mathrm{B}(m, n) \quad(m \neq 0)$ case:

$$
\begin{aligned}
& \alpha_{1} \\
& \bigcirc-\cdots-\alpha_{n}
\end{aligned} \overline{-1}_{-1}^{\alpha_{m+n}}
$$



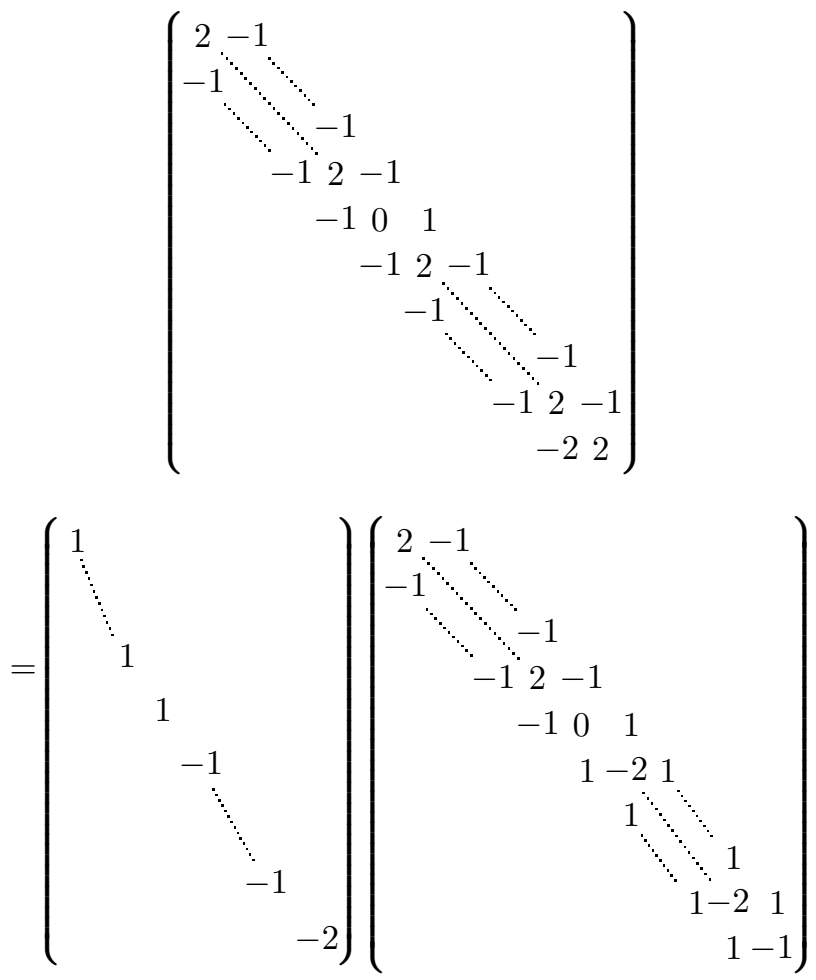

$$
\begin{aligned}
& \Delta_{\overline{0}}^{+}=\left\{\epsilon_{i} \pm \epsilon_{j}, \epsilon_{i^{\prime}}, \delta_{k} \pm \delta_{l}, 2 \delta_{k^{\prime}} \mid 1 \leq i<j \leq m, 1 \leq i^{\prime} \leq m\right. \\
& \left.1 \leq k<l \leq n, 1 \leq k^{\prime} \leq n\right\} \text {. } \\
& \Delta_{\overline{1}}^{+}=\left\{\delta_{i}, \delta_{k} \pm \epsilon_{l}, \mid 1 \leq i, k \leq n, 1 \leq l \leq m\right\} \\
& \Pi=\left\{\delta_{1}-\delta_{2}, \cdots, \delta_{n-1}-\delta_{n}, \delta_{n}-\epsilon_{1}, \epsilon_{1}-\epsilon_{2}, \cdots, \epsilon_{m-1}-\epsilon_{m}, \epsilon_{m}\right\},
\end{aligned}
$$

where $\left\{\epsilon_{i}, \delta_{j} \mid 1 \leq i \leq m, 1 \leq j \leq n\right\}$ is an orthogonal basis such that $(m \neq 0)$, $\left(\epsilon_{i}, \epsilon_{i}\right)=-1$ and $\left(\delta_{j}, \delta_{j}\right)=1$.

3. $\mathrm{B}(0, n)$ case:

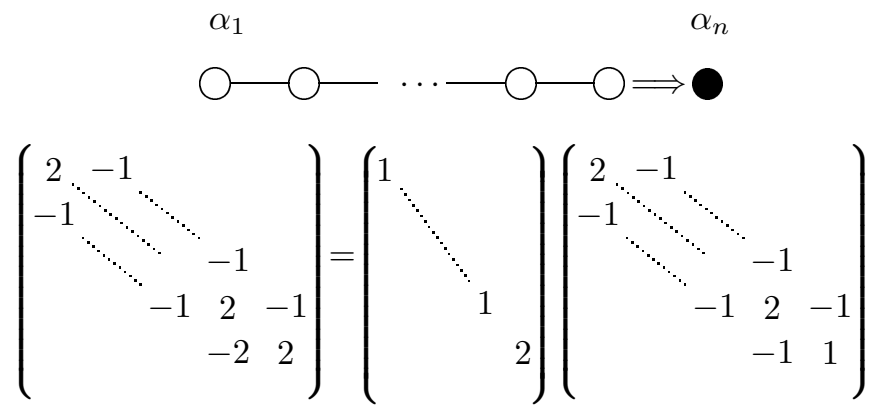


Vol. 76 (2001)

$$
\begin{aligned}
& \Delta_{\overline{0}}^{+}=\left\{\delta_{i} \pm \delta_{j}, 2 \delta_{i^{\prime}} \mid 1 \leq i<j \leq n, 1 \leq i^{\prime} \leq n\right\} . \\
& \Delta_{\overline{1}}^{+}=\left\{\delta_{i} \mid 1 \leq i \leq n\right\} . \\
& \Pi=\left\{\delta_{1}-\delta_{2}, \cdots, \delta_{n-1}-\delta_{n}, \delta_{n}\right\},
\end{aligned}
$$

where $\left\{\delta_{i} \mid 1 \leq i \leq n\right\}$ is an orthogonal basis such that $\left(\delta_{j}, \delta_{j}\right)=1$.

4. $\mathrm{C}(n)$ case:

$$
\begin{aligned}
& \alpha_{1} \\
& \otimes- \\
& \alpha_{n}
\end{aligned}
$$

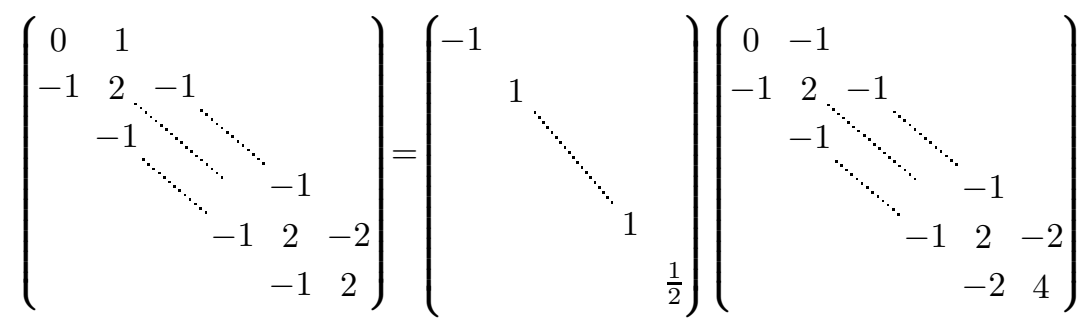

$$
\begin{aligned}
& \Delta_{\overline{0}}^{+}=\left\{\delta_{i} \pm \delta_{j}, 2 \delta_{i^{\prime}} \mid 1 \leq i<j \leq n-1,1 \leq i^{\prime} \leq n-1\right\} . \\
& \Delta_{\overline{1}}^{+}=\left\{\epsilon_{1} \pm \delta_{i} \mid 1 \leq i \leq n-1\right\} . \\
& \Pi=\left\{\epsilon_{1}-\delta_{1}, \delta_{1}-\delta_{2}, \cdots, \delta_{n-2}-\delta_{n-1}, 2 \delta_{n-1}\right\}
\end{aligned}
$$

where $\left\{\epsilon_{1}, \delta_{i} \mid 1 \leq i \leq n-1\right\}$ is an orthogonal basis such that $\left(\epsilon_{1}, \epsilon_{1}\right)=-1$ and $\left(\delta_{j}, \delta_{j}\right)=1$.

5. $\mathrm{D}(m, n)$ case:

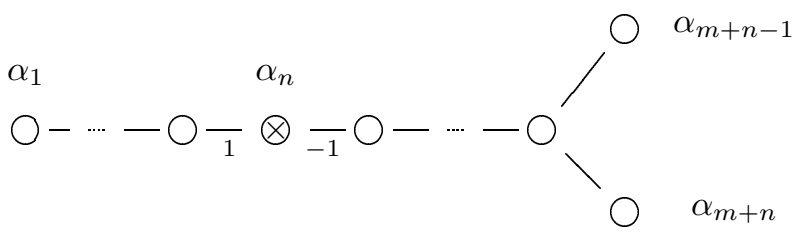



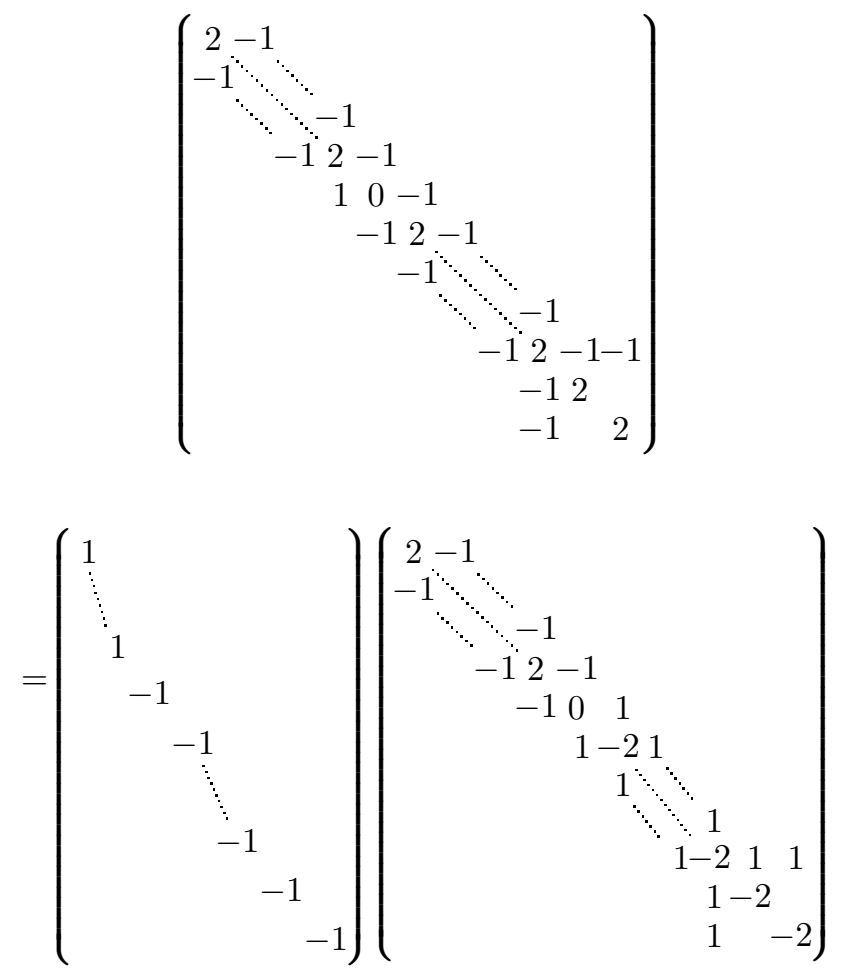

$$
\begin{aligned}
& \Delta_{\overline{0}}^{+}=\left\{\epsilon_{i} \pm \epsilon_{j}, \delta_{k} \pm \delta_{l}, 2 \delta_{k^{\prime}} \mid 1 \leq i<j \leq m, 1 \leq k<l \leq n, 1 \leq k^{\prime} \leq n\right\} . \\
& \Delta_{\overline{1}}^{+}=\left\{\delta_{i} \pm \epsilon_{j}, \mid 1 \leq i \leq n, 1 \leq j \leq m\right\} . \\
& \Pi=\left\{\delta_{1}-\delta_{2}, \cdots, \delta_{n-1}-\delta_{n}, \delta_{n}-\epsilon_{1}, \epsilon_{1}-\epsilon_{2}, \cdots, \epsilon_{m-1}-\epsilon_{m}, \epsilon_{m-1}+\epsilon_{m}\right\},
\end{aligned}
$$

where $\left\{\epsilon_{i}, \delta_{j} \mid 1 \leq i \leq m, 1 \leq j \leq n\right\}$ is an orthogonal basis such that $\left(\epsilon_{i}, \epsilon_{i}\right)=$ -1 and $\left(\delta_{j}, \delta_{j}\right)=1$.

6. $\mathrm{D}(2,1 ; a)$ case: $\quad \alpha_{1} \quad \alpha_{2} \quad \alpha_{3}$

$$
\bigcirc \frac{-1}{1} \otimes-
$$

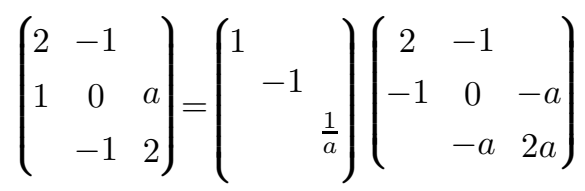




$$
\begin{aligned}
& \Delta_{\overline{0}}^{+}=\left\{2 \epsilon_{1}, 2 \epsilon_{2}, 2 \epsilon_{3}\right\} . \\
& \Delta_{\overline{1}}^{+}=\left\{\epsilon_{2} \pm \epsilon_{1} \pm \epsilon_{3}\right\} . \\
& \Pi=\left\{2 \epsilon_{1}, \epsilon_{2}-\epsilon_{1}-\epsilon_{3}, 2 \epsilon_{3}\right\},
\end{aligned}
$$

where $\left\{\epsilon_{1}, \epsilon_{2}, \epsilon_{3}\right\}$ is an orthogonal basis such that $\left(\epsilon_{1}, \epsilon_{1}\right)=\frac{1}{2},\left(\epsilon_{2}, \epsilon_{2}\right)=$ $-\frac{1}{2} a-\frac{1}{2}$ and $\left(\epsilon_{3}, \epsilon_{3}\right)=\frac{1}{2} a$.

7. $\mathrm{F}(4)$ case:

$$
\begin{aligned}
& \alpha_{1} \quad \alpha_{2} \quad \alpha_{3} \quad \alpha_{4} \\
& \otimes \frac{1}{-} \bigcirc \Leftarrow \bigcirc-\bigcirc
\end{aligned}
$$

$$
\begin{aligned}
& \left(\begin{array}{cccc}
0 & 1 & & \\
-1 & 2 & -2 & \\
& -1 & 2 & -1 \\
& & -1 & 2
\end{array}\right)=\left(\begin{array}{cccc}
-1 & & \\
& 1 & \\
& \frac{1}{2} & \\
& & \frac{1}{2}
\end{array}\right)\left(\begin{array}{cccc}
0 & -1 & \\
-1 & 2 & -2 & \\
& -2 & 4 & -2 \\
& & -2 & 4
\end{array}\right) \\
& \Delta_{\overline{0}}^{+}=\left\{\delta_{1},-\epsilon_{i},-\epsilon_{k} \pm \epsilon_{l} \mid 1 \leq i \leq 3,1 \leq k<l \leq 3\right\} . \\
& \Delta_{\overline{1}}^{+}=\left\{\frac{1}{2}\left(\delta_{1} \pm \epsilon_{1} \pm \epsilon_{2} \pm \epsilon_{3}\right)\right\} . \\
& \Pi=\left\{\frac{1}{2}\left(\delta_{1}+\epsilon_{1}+\epsilon_{2}+\epsilon_{3}\right),-\epsilon_{1}, \epsilon_{1}-\epsilon_{2}, \epsilon_{2}-\epsilon_{3}\right\},
\end{aligned}
$$

where $\left\{\delta_{1}, \epsilon_{1}, \epsilon_{2}, \epsilon_{3}\right\}$ is an orthogonal basis such that $\left(\epsilon_{i}, \epsilon_{i}\right)=2$ and $\left(\delta_{1}, \delta_{1}\right)=$ $-6$

8. $\mathrm{G}(3)$ case:

$$
\begin{gathered}
\alpha_{1} \quad \alpha_{2} \quad \alpha_{3} \\
\otimes \frac{1}{\frac{1}{2}} \bigcirc \Leftarrow \bigcirc \\
\left(\begin{array}{ccc}
0 & \frac{1}{2} & \\
-1 & 2 & -3 \\
& -1 & 2
\end{array}\right)=\left(\begin{array}{ccc}
-\frac{1}{2} & \\
& 1 \\
& \frac{1}{3}
\end{array}\right)\left(\begin{array}{ccc}
0 & -1 & \\
-1 & 2 & -3 \\
& -3 & 6
\end{array}\right) \\
\Delta_{\overline{0}}^{+}=\left\{2 \delta_{1},-\epsilon_{1}, \epsilon_{2}, \epsilon_{3}, \epsilon_{i}-\epsilon_{j} \mid 1 \leq i<j \leq 3\right\} . \\
\Delta_{\overline{1}}^{+}=\left\{\delta_{1}, \delta_{1} \pm \epsilon_{i} \mid 1 \leq i \leq 3\right\} . \\
\Pi=\left\{\delta_{1}+\epsilon_{1}, \epsilon_{2},-\epsilon_{2}+\epsilon_{3}\right\},
\end{gathered}
$$

where $\left\{\delta_{1}, \epsilon_{1}, \epsilon_{2}, \epsilon_{3}\right\}$ are elements of $\left(\bigoplus_{i} \mathbb{C} \epsilon_{i} \oplus \mathbb{C} \delta_{1}\right) / \mathbb{C}\left(\epsilon_{1}+\epsilon_{2}+\epsilon_{3}\right)$, such that $\left(\epsilon_{i}, \epsilon_{i}\right)=2,\left(\epsilon_{i}, \epsilon_{j}\right)=-1 \quad(i \neq j),\left(\epsilon_{i}, \delta_{1}\right)=0$ and $\left(\delta_{1}, \delta_{1}\right)=-2$. 


\section{References}

[Che] C. Chevalley, Sur certains groupes simples, Tohoku Math. J. 7(2) (1955), 14-66.

[Gar] H. Garland, The arithmetic theory of loop groups, Publ. I.H.E.S. 52 (1980), 5-136.

[Kac1] V. G. Kac, Lie superalgebras, Advances in Math. 26 no. 1 (1977), 8-96.

[Kac2] V. G. Kac, Representations of classical Lie superalgebras, Lect. Note in Math. 676 (1978), 597-626.

[Kas] C. Kassel, Kähler differentials and coverings of complex simple Lie algebras extended over a commutative algebra, J. Pure Appl. Algebra 34 no. 2-3 (1984), 265-275.

[KL] C. Kassel, J.-L. Loday, Extensions centrales d'algèbres de Lie, Ann. Inst. Fourier 32 no. 4 (1982), 119-142.

$[\mathrm{KW}]$ V. G. Kac, M. Wakimoto, Integrable highest weight modules over affine superalgebras and number theory, Lie theory and geometry, 415-456, Progr. Math. 123, Birkhäuser Boston, Boston, MA, 1994.

[Lod] J.-L. Loday, Cyclic homology, Second edition. Grundlehren der Mathematischen Wissenschaften, 301. Springer-Verlag, Berlin, 1998.

[MP] R. V. Moody, A. Pianzola, Lie Algebras with Triangular Decomposition, Can. Math. Soc., John Wiley \& Sons, 1995.

[Ya] H. Yamane, On defining relations of affine Lie superalgebras and affine quantized universal enveloping superalgebras, Publ. RIMS, vol 35 no. 3 (1999), 321-390.

Kenji Iohara

Department of Mathematics

Faculty of Science

Kobe University

Kobe 657-8501

Japan

e-mail: iohara@math.kobe-u.ac.jp
Yoshiyuki Koga

Department of Mathematics

Faculty of Science

Osaka University

Osaka 560-0043

Japan

e-mail: koga@math.sci.osaka-u.ac.jp

(Received: April 21, 2000) 